\title{
TWO NEW SCREE PLANT COMMUNITIES IN THE TRIGLAV MOUNTAINS (JULIAN ALPS, SLOVENIA) DVE NOVI RASTLINSKI ZDRUŽBI MELIŠČ V TRIGLAVSKEM POGORJU (JULIJSKE ALPE, SLOVENIJA)
}

\author{
Igor DAKSKOBLER* \& Branko ZUPAN**
}

http://dx.doi.org/10.3986/fbg0018

\begin{abstract}
Two new scree plant communities in the Triglav Mountains (Julian Alps, Slovenia)

In the Triglav Mountains, mainly on the slopes and plateaus to the west of Mt. Triglav (Glava v Zaplanji, Vrh Zelenic) we found new localities of three rare species of Slovenian flora, Crepis terglouensis, Cerastium uniflorum and Geum reptans, and made a phytosociological inventory of their sites. Based on comparisons with similar communities within which they occur elsewhere in the Eastern and Southeastern Alps we described two new associations, Crepido terglouensis-Potentilletum nitidae (alliance Thlaspion rotundifolii) and Saxifrago carniolicae-Cerastietum uniflorae (alliance Arabidion caeruleae).

Key words: Alpine flora, phytosociology, synsystematics, Crepis terglouensis, Cerastium uniflorum, Geum reptans, Triglav National Park, Slovenia
\end{abstract}

\section{IZVLEČEK}

Dve novi rastlinski združbi melišč v Triglavskem pogorju (Julijske Alpe, Slovenija)

V Triglavskem pogorju, predvsem na pobočjih in planotah zahodno od Triglava (Glava v Zaplanji, Vrh Zelenic) smo našli nova nahajališča treh redkih vrst v flori Sloveniji, Crepis terglouensis, Cerastium uniflorum in Geum reptans in fitocenološko popisali njihova rastišča. Na podlagi primerjav s podobnimi združbami, v katerih uspevajo omenjene vrste drugod v Vzhodnih in Jugovzhodnih Alpah, smo opisali dve novi asociaciji Crepido terglouensis-Potentilletum nitidae (zveza Thlaspion rotundifolii) in Saxifrago carniolicae-Cerastietum uniflorae (zveza Arabidion caeruleae).

Ključne besede: alpska flora, fitocenologija, sinsistematika, Crepis terglouensis, Cerastium uniflorum, Geum reptans, Triglavski narodni park, Slovenija

\footnotetext{
* Institute of Biology, Scientific Research Centre of the Slovenian Academy of Sciences and Arts, Regional unit Tolmin, Brunov drevored 13, SI-5220 Tolmin and Biotechnical Faculty of the University in Ljubljana, Department of Forestry and Renewable Forest Resources, Večna pot 83, SI-1000 Ljubljana, Igor.Dakskobler@zrc-sazu.si

** Savica 6, SI-4264 Bohinjska Bistrica
} 


\section{INTRODUCTION}

When mapping the flora on the slopes and plateaus west of Mt. Triglav in 2015 and 2016 we found and recorded fascinating scree communities with three rare species of Slovenian flora - Crepis terglouensis, Cerastium uniflorum and Geum reptans. We compared them to similar communities that had been studied in the Triglav Mountains and in the Julian Alps by T. WRABER (1972) and with similar communities elsewhere in the Eastern Alps, and classified them into a syntaxonomic system.

\section{METHODS}

Alpine communities under Mt. Triglav were studied applying the Braun-Blanquet method (BRAUN-BLANQUET 1964). A total of 29 relevés (of which five had already been published by T. Wraber, 1972 and one by the authors of this paper - DAKsKobler \& SURINA, 2017) were entered into the FloVegSi database (Fauna, Flora, Vegetation and Paleovegetation of Slovenia) of the Jovan Hadži Institute of Biology at SRC SASA (T. SelišKar, VReš et A. SelišKar 2003). They were arranged into a working table based on hierarchical classification. We transformed the combined cover-abundance values with numerical values (1-9) according to van der MAAREL (1979). Numerical comparisons were performed with the SYN-TAX 2000 program package (PoDANi 2001). The relevés were compared by means of (unweighted) average linkage method - UPGMA, using Wishart's similarity ratio.

In the first step we used numerical analyses as the basis on which we formed floristically homogeneous groups of relevés that were subsequently compared, using the same methodology, with similar communities in the Eastern Alps, also using hierarchical classification and the same method as when we compared individual relevés.
The nomenclature sources for the names of vascular plants are the Mala flora Slovenije (MARTinčIč et al. 2007) and Flora alpina (Aeschimann et al. 2004a,b). Prof. Andrej Martinčič determined the collected mosses. For the names of syntaxa we follow ENGLISCH et al. (1993), Theurillat (2004), Šilc \& Čarni (2012), E. Pignatti \& S. Pignatti (2014) and Mucina et al. (2016). In the classification of species into phytosociological groups (groups of diagnostic species) we mainly refer to the Flora alpina (AEschimann et al. 2004a,b). The geographic coordinates of relevés are determined according to the Slovenian geographic coordinate system D 48 (5th zone) on the Bessel ellipsoid and with Gauss-Krüger projection.

The relevés discussed in this article were made in the Triglav range of the Julian Alps, mostly on the plateuas west and southwest of Triglav. The geological bedrock in the study area is mainly calcareous, limestone and dolomite limestone (BUSER 2009). The studied communities occur on initial soils (lithosols) - LoVRENČAK (1998), VIDIC et al. (2015). The climate is cold and humid, with mean annual precipitation of 2,500 to $3,000 \mathrm{~mm}$ (ZuPANČIČ 1998) and mean annual air temperature of $-2{ }^{\circ} \mathrm{C}$ to $0^{\circ} \mathrm{C}$

\section{RESULTS AND DISCUSSION}

\subsection{Review of the studied syntaxa, with types of newly described communities}

Thlaspietea rotundifolii Br.-Bl. 1948

Thlaspietalia rotundifolii $\mathrm{Br}$.-Bl. in Br.-Bl. et Jenny 1926

Thlaspion rotundifolii Jenny-Lips 1930

Papaveri julici-Thlaspietum rotundifolii T. Wraber 1970 Crepidetum terglouensis Seibert 1977

Crepido terglouensis-Potentilletum nitidae ass. nov. hoc. loco, the nomenclature type, holotypus, is relevé 12 in Table 1. -typicum, subass. nov., the nomenclature type is the same as the nomenclature type of the association -caricetosum firmae, the nomenclature type, holotypus, is relevé 5 in Table 1.

Saxifrago sedoidis-Geumetum reptantis nom. prov. Arabidetalia caeruleae Rübel ex Nordhagen 1937 Arabidion caeruleae Br.-Bl. In Br.-Bl. et Jenny 1926 Saxifrago carniolicae-Cerastietum uniflorae ass. nov. hoc loco, the nomenclature type, holotypus, is relevé 18 in Table 1.

Asplenietea trichomanis (Br.-Bl. in Meier et Br.-Bl. 1934) Oberd. 1977 
Potentilletalia caulescentis Br.-Bl. in Br.-Bl. et Jenny 1926

Physoplexido comosae-Saxifragion petraeae Mucina et Theurillat 2015 (syn. Androsaco-Drabion to- mentosae T. Wraber 1970, Phyteumato-Saxifragion petraeae Mucina in Šilc et Čarni 2012)

Potentilletum nitidae Wikus 1959

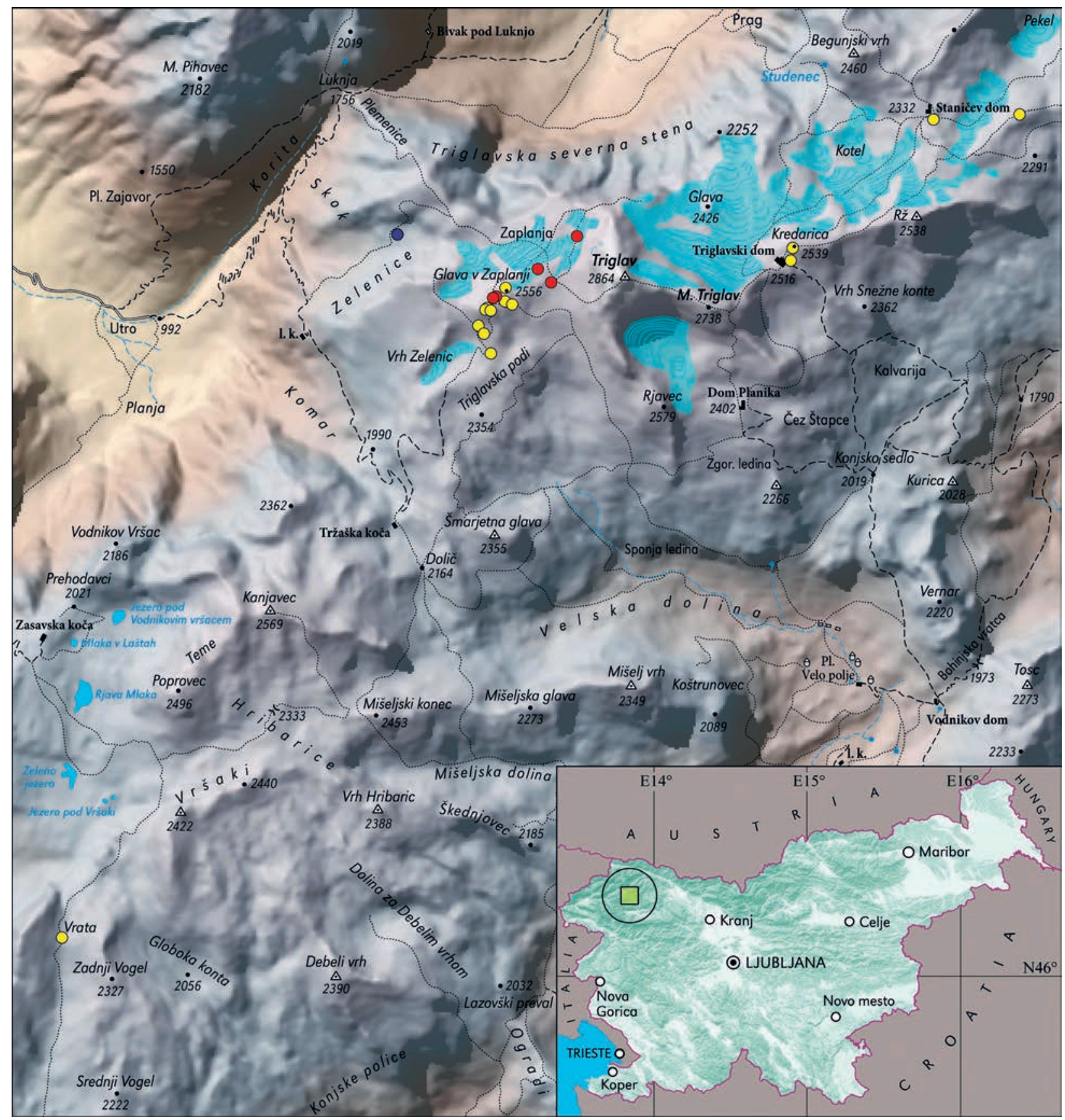

Figure 1: Localities of recorded stands in the Triglav mountains; yellow circle - stands of the association Crepido terglouensisPotentilletum nitidae, red circle - stands of the association Saxifrago-Cerastietum uniflorae, blue circle - stand of the association Saxifrago-Geumetum reptantis

Slika 1: Nahajališča preučenih sestojev v Triglavskem pogorju: rumen krog - sestoji asociacije Crepido terglouensis-Potentilletum nitidae, rdeč krog - sestoji asociacije Saxifrago-Cerastietum uniflorae, moder krog - sestoj asociacije Saxifrago-Geumetum reptantis 


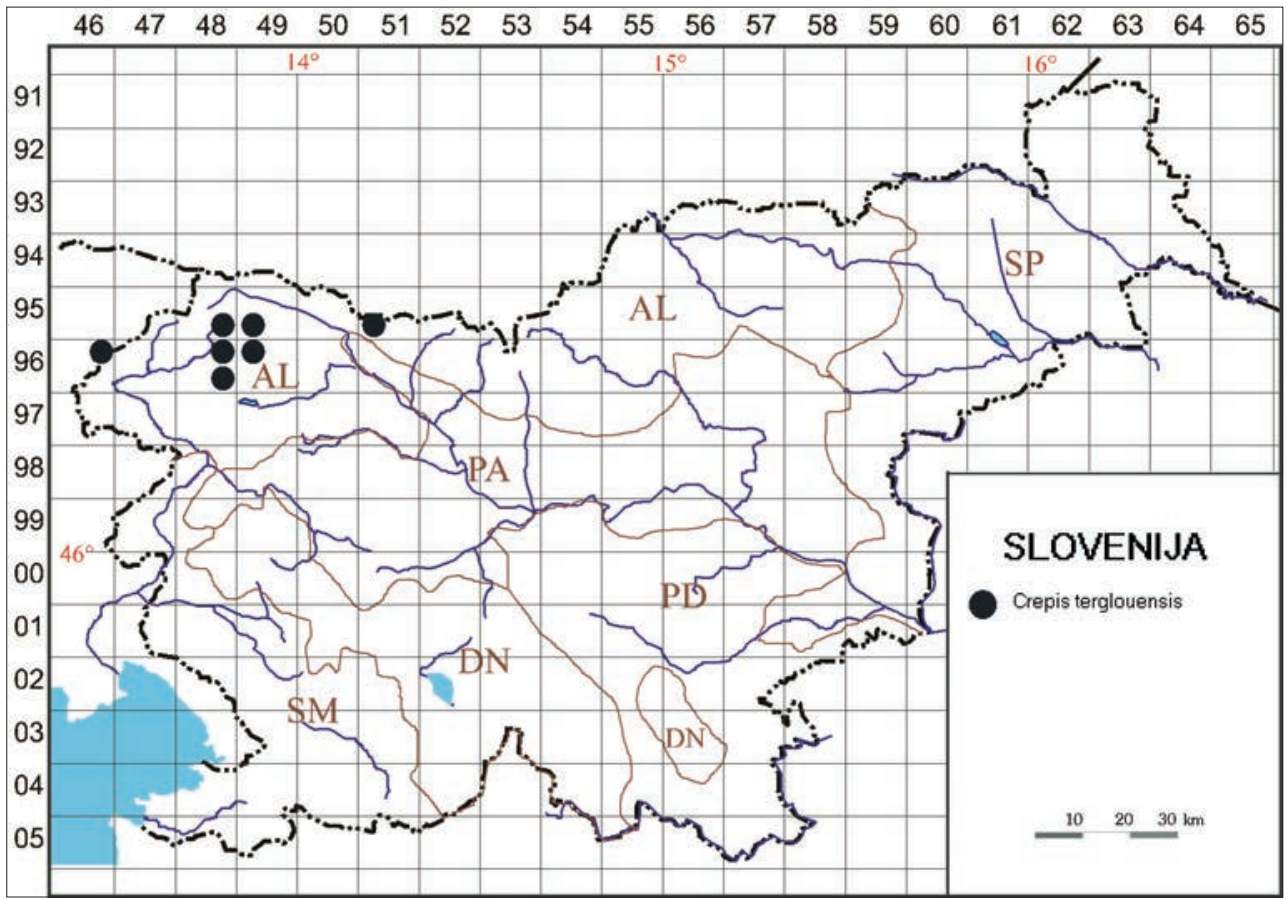

Figure 2: Distribution of Crepis terglouensis in Slovenia Slika 2: Razširjenost vrste Crepis terglouensis v Sloveniji

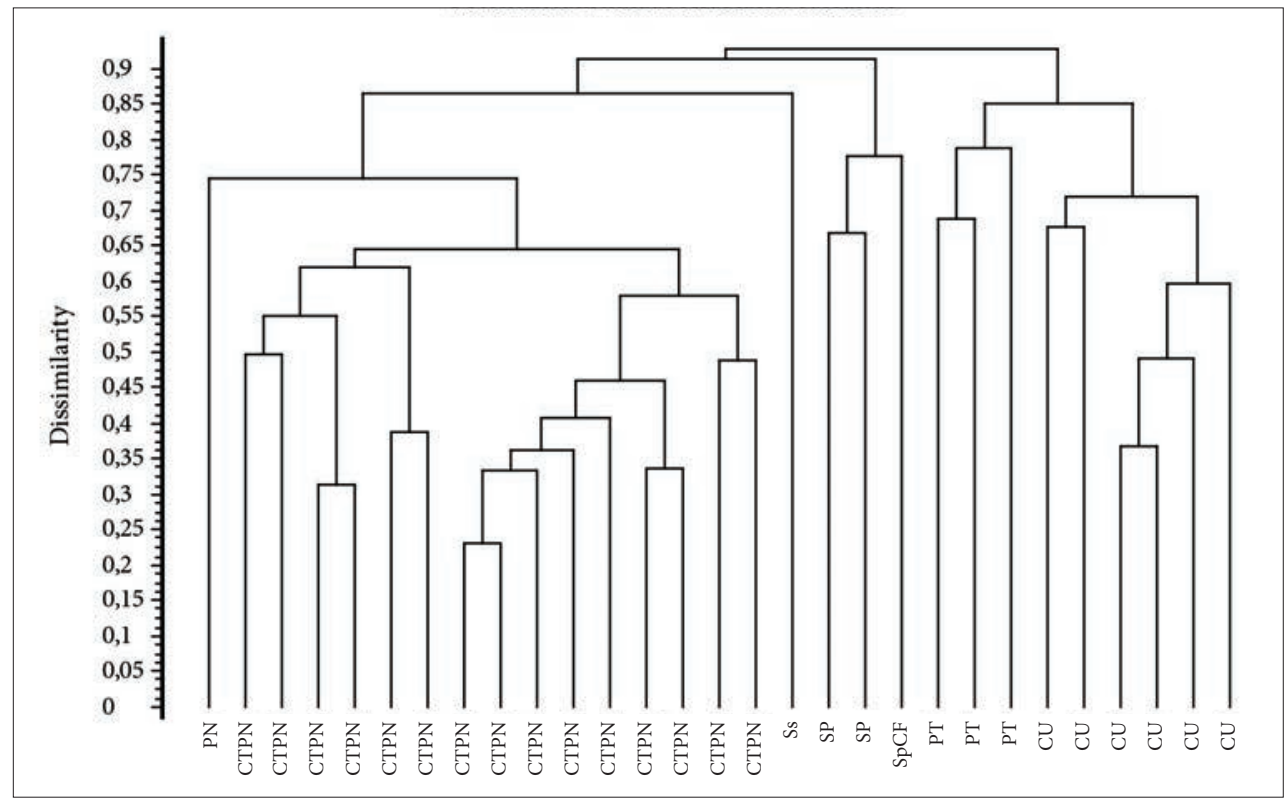

Figure 3: Dendrogram of recorded stands in the Triglav Mountains, UPGMA, 1- similarity ratio PN Potentilletum nitidae; CTPN Crepido terglouensis-Potentilletum nitidae, Ss Salicetum serpyllifoliae nom. prov., SP Saxifragetum paniculatae nom. prov., SpCF Saxifrago paniculatae-Caricetum fuliginosae, PT Papaveri julici-Thlaspietum rotundifolii, CU Saxifrago-Cerastietum uniflorae

Slika 3: Dendrogram popisov preučenih sestojev v Triglavskem pogorju, UPGMA, komplement Wishartovega koeficienta podobnosti 


\subsection{Crepis terglouensis and its communities in Slovenia}

According to Flora alpina (Aeschimann et al. 2004b: 668) Crepis terglouensis is an eastern-Alpine species, a character species of the alliance Thlaspion rotundifolii that comprises vegetation of subalpine-alpine calcareous screes. The species also gave its name to the alpine community Crepidetum terglouensis Seibert 1977 from the Eastern Alps, which was presented with a phytosociological table by EgGeNSBERger (1994: 64-66, 7677). Certain similarities with this community can be observed also in the relevé published by SutTer (1969: 353) that he had made together with T. Wraber under the Planika Lodge at Mt. Triglav. T. Wraber (1972, 1990) recorded Crepis terglouensis mainly in the stands of a special form of the association Papaveri julici-Thlaspietum rotundifolii. He found it on two screes at Kredarica and on Grlo pass between Oltar and Dovški Križ. E. \& S. Pignatti $(2014,2016)$ mention it as a rare species in the stands of two other alpine scree communities, Leontodontetum montani and Papaveretum rha- etici, and in the special form (Seleginella-Homogyne) of the association Seslerio-Caricetum sempervirentis. A phytosociological inventory of its two new localities in the Slovenian Alps (on talus under Prestreljenik in the Kanin Mountains - Praprotnik, 1997, 2002, and on the scree under Mt. Stol in the Karavanke Mts. Nova K 2015) has not been made until now. Novak (ibid.) mentions companion species Sesleria caerulea and Campanula cochleariifolia for the locality under Mt. Stol. In our research we made most of the relevés with Crepis terglouensis on fine talus on Glava nad Zaplanjo under Mt. Triglav and on the neighbouring slopes towards Dolič (9648/2), but we also found a new locality on a similar site on Vrata pass between Zelnarica and Zadnji Vogel (9648/4) at the elevation of 2,192 m (leg. \& det. I. Dakskobler, B. Anderle and B. Zupan, 23. 8. 2016, herbarium LJS), which is a new locality of this species in the new quadrant (Figures 1,2). In the comparison of our relevés with Crepis terglouensis and (or) Cerastium uniflorum with similar relevés made by T. Wraber (1972), the relevés with co-dominating Potentilla nitida and Crepis terglouensis grouped

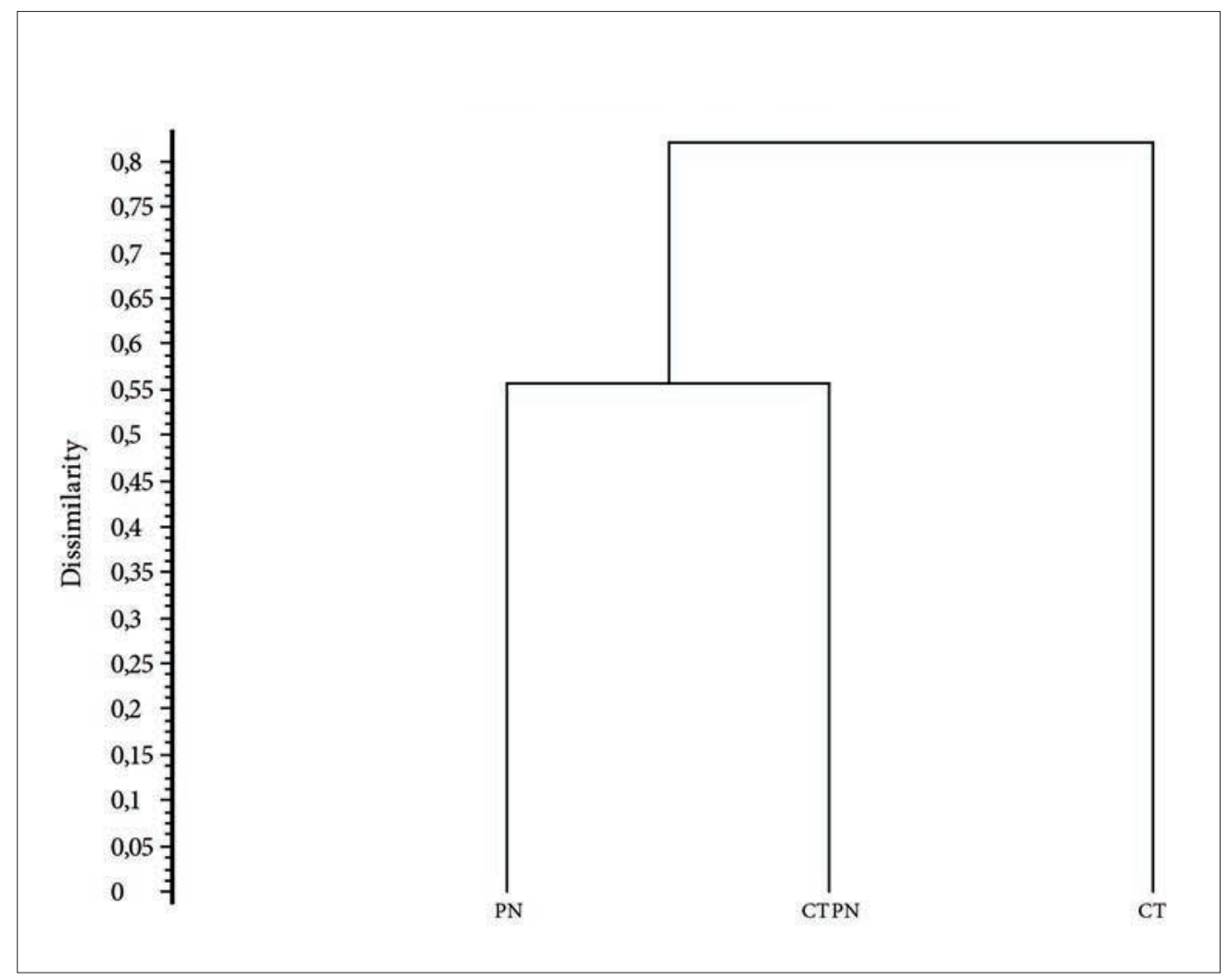

Figure 4: Dendrogram of syntaxa Potentilletum nitidae (PN), Crepidetum terglouensis (CT) and Crepido terglouensis-Potentilletum nitidae (CTPN), UPGMA, 1- similarity ratio Slika 4: Dendrogram sintaksonov Potentilletum nitidae (PN), Crepidetum terglouensis (CT) and Crepido terglouensis-Potentilletum nitidae (CTPN), UPGMA, komplement Wishartovega koeficienta podobnosti 
separately (Figure 3). We obviously inventoried a form of alpine vegetation that is transitional between scree communities, chasmophytic communities and stony grasslands, which had already been demonstrated by Sutter's relevé (ibid.) and mentioned also by EgGensBERGER (ibid.). We therefore made a synthetic table (Appendix 1) in which we compared 15 relevés of the studied stands with 33 relevés of the association Potentilletum nitidae (T. Wraber 1972, Table 3) and with 25 relevés of the association Crepidetum terglouensis (Eggensberger 1994, Table 6, columns 85-111). The results (Figure 4) indicate that our relevés are more similar to the stands of the association Potentilletum nitidae. They could therefore be classified into the new subassociation Potentilletum nitidae crepidetosum terglouensis, but the analysis of diagnostic species (Table 2 , column 1) indicates the predominance of scree species from the order Thlaspietelia rotundifolii (37.43\%) over the diagnostic chasmophytic species from the order Potentilletalia caulescentis (23.8\%). Most of the relevés were made on fine talus that is almost consistently mixed with well-decomposed humus (mull), on levelled terrain with a relatively persistent snow cover.
As a rule, Potentilla nitida is the dominant species in inventoried stands and its joint occurrence with the characteristic scree species Crepis terglouensis on relatively small but ecologically unique areas between rocks (fine talus) can indicate a stage in development (succession) that could be partly associated with the ongoing climate change, reduced precipitation volumes and shorter periods of snow cover on the plateaus to the west of Mt. Triglav, as demonstrated by the measurements of the Triglav Glacier at a similar elevation (GABRovec et al. 2014). This stage in succession can be treated also at the rank of the new association Crepido terglouensis-Potentilletum nitidae, which is classified into the alliance Thlaspion rotundifolii. Diagnostic species of the new association are Potentilla nitida, Crepis terglouensis, Alyssum ovirense, Eritrichium nanum and Minuartia cherlerioides. We distinguish between two subassociations, typical (-typicum) and-caricetosum firmae. The differential species of the latter are Carex firma, Silene acaulis and Salix retusa, which might indicate a transition towards stony alpine grasslands from the association Gentiano terglouensis-Caricetum firmae.

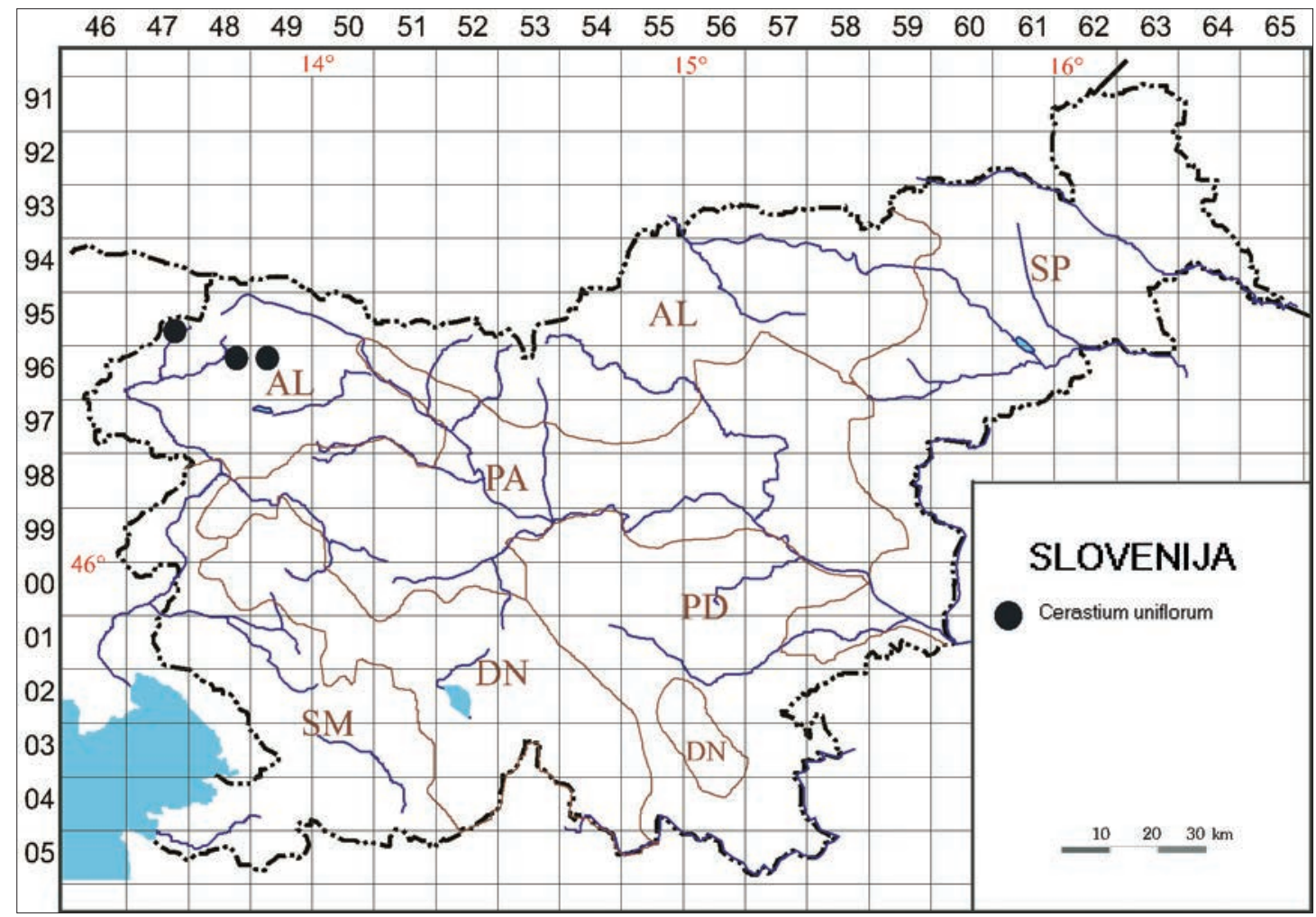

Figure 5: Distribution of Cerastium uniflorum in Slovenia Slika 5: Razširjenost vrste Cerastium uniflorum v Sloveniji 


\subsection{Cerastium uniflorum and its communities in Slovenia}

Cerastium uniflorum is an Alpine-Carpathian species, a character species of silicate subalpine and alpine screes (alliance Androsacion alpinae ) - AEsCHIMANN et al. (2004a: 298). Its distribution in Slovenia is shown in Figure 5. Its reported localities are only on Mt. Triglav and its vicinity, on Mt. Rjavina and on Mt. Mangart. T. WrABER (1990: 130, 1972) reported its occurrence in the stands of associations Potentilletum nitidae and Papaveri julici-Thlaspietum rotundifolii. E. \& S. Pignatti $(2014,2016)$ recorded this species in the stands of associations Drabetum hoppeanae, Saxifragetum sedoidis, Leontodontetum montani, Papaveretum rhaetici, Festucetum pulchellae, Vitaliano-Eritrichetum nani, Arabidetum caeruleae, Saxifragetum bryoidis and several others. In our relevés it occurs with individual specimens within associations Saxifrago paniculatae-Caricetum fuliginosae (DAKskobler \& Surina 2017) and Crepido-Potentilletum nitidae (this article), but it is also one of the dominating species in six relevés on mainly fine talus and on sites with a persistent snow cover (Figure 1, relevés 17-21 in Table 1). These relevés grouped separately from other relevés of alpine screes and alpine swards on rock ledges (Figure 3). The most frequent species in addition to Cerastium uniflorum is Saxifrage sedoides, so we compared them with the association Saxifragetum sedoidis from the Dolomites (E. \& S. Pignatti 2016: 204: Association table 9.2, 406407) - Appendix 2. Surina (2005) prepared a phytosociological table for the Krn Mts. in which he presented a similar syntaxon Saxifragetum stellaro-sedoidis var. geogr. Ranunculus traunfellneri, but in his stands he did not record Cerastium uniflorum, which is absent also from the original description of this association (ENGLisch 1999). Floristic similarity of our relevés with the relevés of the stands of the association Saxifragetum sedoidis is only 41\% (SøRENSEN 1948), which does not allow for its classification into this association. These stands can also be explained as a successional stage, because in the extreme conditions of the alpine belt Cerastium uniflorum can grow on various sites and overgrows even areas where it may not have occurred 50 or 100 years ago. For this reason and based on the composition of diagnostic species (Table 2, column 2) we classify them into the new association Saxifrago carniolicae-Cericetum uniflorae and into the alliance Arabidion caeruleae. Its diagnostic species are Cerastium uniflorum, Saxifraga sedoides and Saxifraga exarata subsp. carniolica. The latter is endemic to the Southeastern Alps and a character species of alpine chasmophytic communities (Potentilletum nitidae, Potentillo clusianae-Campanuletum zoysii) - T. WRABER (2006: 70) and of stony grasslands from alliances Caricion firmae and Seslerion variae (AESCHIMANN et al. 2004: 2010). The first two listed species characterise an alpine community on fine talus in areas with longlasting snow cover and the third characterises the new association mainly in terms of phytogeography and partly indicates similar development of communities on fine gravel with communities on stony alpine swards and rock crevices. Abundant Sagina saginoides in one of the stands indicates locally improved soil conditions with an abundance of fine weathered material (mull).

\subsection{Geum reptans in the Triglav Mountains}

Aeschimann et al. (2004a: 758) classify the SouthEuropean montane species Geum reptans as a character species of the alliance Androsacion alpinae. The only reported localities in the Julian Alps so far have been those on Mts. Mangart and Kanjavec (T. WRABer 2006, ZupAN \& DAKSKobler 2007, Figure 6). On 8 August 2016 we found a new locality of this scree species on the western slopes of Triglav, in the cirque under Vrh Zelenic, on the elevation of 2,060 m (DAKsкobler 2017, Figure 1). The site is a well-overgrown, consolidated scree with rocks of various sizes. Its species composition is shown in Column 2 of Table 3. Column 1 in the same table presents the releve of the community on the locality at Teme between Kanjavec and Poprovec (Zupan \& DAKsKobler 2007), which was identified as a transition between the community of Potentilletum nitidae and the stand of the association Papaveri julici-Thlaspietum rotundifolii. The stand under Vrh Zelenic cannot be classified into either of the mentioned associations. It characterises a scree community with many species of stony Alpine swards (from the association Gentiano terglouensis-Caricetum firmae). It is provisionally classified into the association Saxifrago sedoidis-Geumetum reptantis nom. prov. and into the alliance Thlaspion rotundifolii. In addition to Geum reptans the species that characterise this community are mainly those from the genus Saxifraga (S. sedoides, S. paniculata, S. aizoides, S. crustata, S. squarrosa) and two typical scree species, Festuca nitida and Poa minor. 


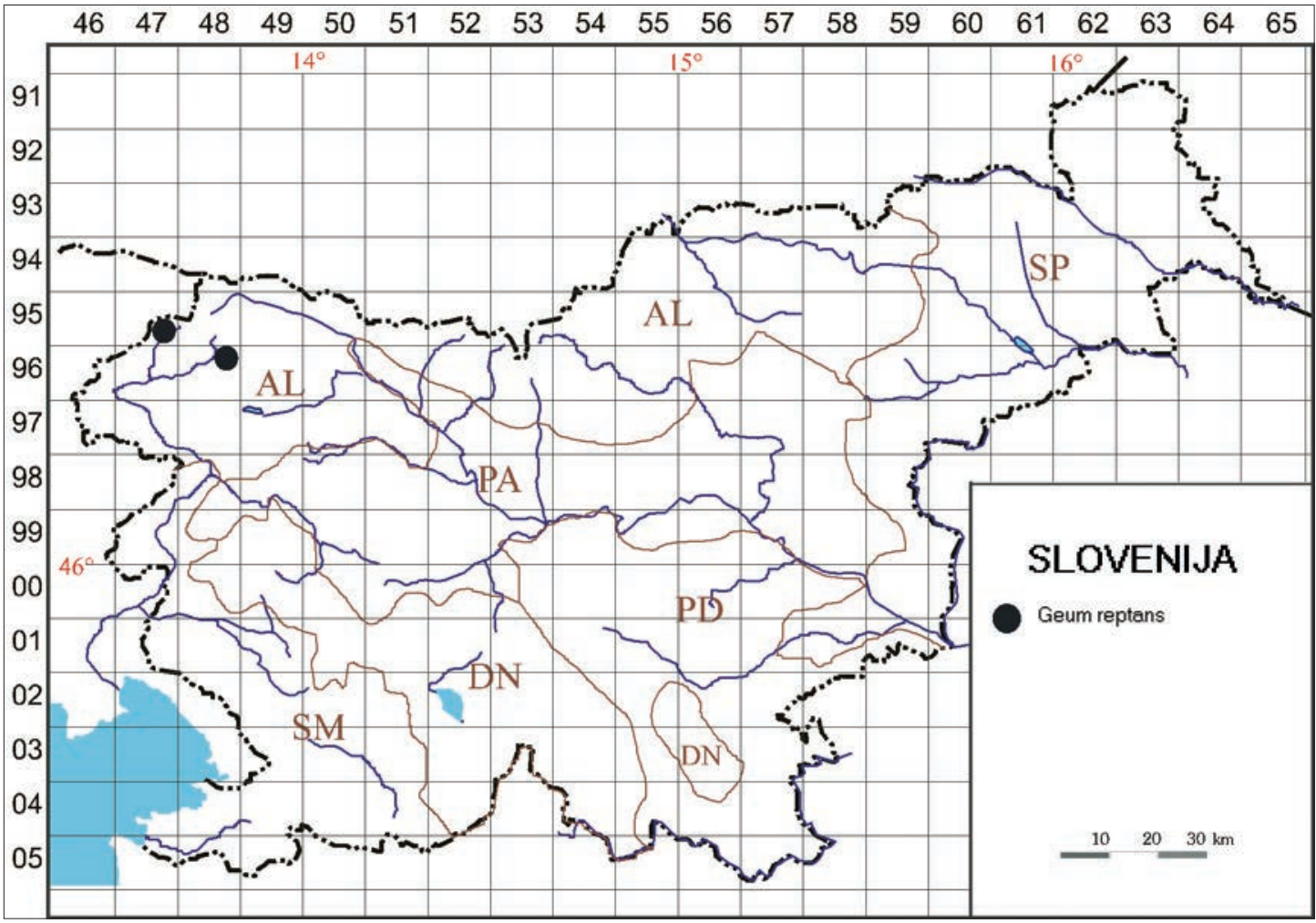

Figure 6: Distribution of Geum reptans in Slovenia Slika 6: Razširjenost vrste Geum reptans v Sloveniji

\section{CONCLUSIONS}

The result of floristic mapping in the western part of Mt. Triglav (Glava v Zaplanji, Vrh Zelenic) and on Vrata pass between Velika Zelnarica and Zadnji Vogal are new localities of three alpine scree and (or) chasmophytic species that are rare in Slovenia and listed on the Red List (ANon. 2002): Cerastium uniflorum (new localities in quadrant 9648/2), Crepis terglouensis (new localities in quadrants 9648/2 and 9648/4) and Geum reptans (new locality in the existing quadrant, 9648/2, new to the flora of Triglav). We made a phytosociological inventory of the communities in which they grow and determined untypical stands on fine talus with fine weathered material (mull) that are transitional between alpine chasmophytic, scree and snow bed communities and could be a successional stage in areas that decades ago were covered by snow for much longer periods than they are today. Based on their comparison with similar communities from the Eastern and Southeastern Alps we described two new associations, Crepido terglouensis-Potentilletum nitidae and Saxifrago carniolicae-Cerastietum uniflorae. Even though their stands are located in the vicinity of quite popular mountain trails to Triglav they are not yet endangered by increasing numbers of tourists in the summer as there are no other interventions into this area. In addition, neither Cerastium uniflorum nor Crepis terglouensis are prominent species and are quite inconspicuous outside their flowering season. The new locality of Geum reptans, which occurs also in the nontypical scree community (Saxifrago sedoidis-Geumetum reptantis nom. prov.), is in a remote pathless area in the cirque under Vrh Zelenic and therefore still outside any direct influence of man. 


\section{POVZETEK}

\subsection{Uvod}

Pri kartiranju flore na pobočjih in planotah zahodno od Triglava smo v letih 2015 in 2016 našli in popisali zanimive meliščne združbe, $\mathrm{v}$ katerih uspevajo tri redke vrste slovenske flore: Crepis terglouensis, Cerastium uniflorum in Geum reptans. Primerjali smo jih s podobnimi združbami, ki jih je v Triglavskem pogorju in v Julijskih Alpah raziskal T. WRABER (1972), in s podobnimi združbami drugod $\mathrm{v}$ Vzhodnih Alpah in jih uvrstili v sintaksonomski sistem.

\subsection{Metode}

Alpinske združbe pod Triglavom smo preučevali po ustaljeni srednjeevropski metodi (BRAUN-BLANQUET 1964). Skupno 29 popisov (od tega jih je pet predhodno objavil že T. WrABER, 1972, enega pa mi DAKSKOBler \& Surina, 2017) smo vnesli v podatkovno bazo FloVegSi (T. SelišKar, Vreš \& A. SElišKar 2003). Fitocenološke popise smo v delovno tabelo uredili na podlagi hierarhične klasifikacije. Kombinirane ocene zastiranja in pogostnosti smo pretvorili v številčne vrednosti (1-9) - van der MAAREL 1979). Popise smo primerjali z metodo kopičenja na podlagi povezovanja (netehtanih) srednjih razdalj - (Unweighted) average linkage method - UPGMA. Uporabljali smo programski paket SYN-TAX 2000 (PODANI 2001) in kot mero različnosti komplement koeficienta "similarity ratio«. V prvem koraku smo na podlagi numeričnih analiz oblikovali floristično homogene skupine popisov, ki smo jih nato z enakim metodološkim pristopom primerjali s podobnimi združbami v Vzhodnih Alpah, pri tem pa prav tako uporabili hierarhično klasifikacijo in isto metodo kot pri primerjavi posamičnih popisov.

Nomenkalturna vira za imena prarotnic in semenk sta Mala flora Slovenije (MARTINČıč et al. 2007) in Flora alpina (Aeschimann et al. 2004a,b). Mahove, ki v preučenih združbah nimajo večjega zastiranja, je določil prof. Andrej Martinčič. Nomenklaturni viri za imena sintaksonov so ENGLISCH et al. (1993), THEURILlat (2004), Šilc \& ČArni (2012), E. Pignatti \& S. PiGnatti (2014) in Mucina et al. (2016). Pri uvrščanju vrst $\mathrm{v}$ fitocenološke skupine smo $\mathrm{v}$ glavnem upoštevali delo Flora alpina (AEschimann et al., ibid.). Geografske koordinate popisov smo določili po slovenskem geografskem koordinatnem sistemu D 48 (cona 5) na Besselovem elipsoidu in z Gauss-Krügerjevo projekcijo.

Popise smo naredili v Triglavskem pogorju v Julijskih Alpah, večino na planotah zahodno in jugozaho- dno od vrha Triglava. Kot geološka podlaga se pojavljata na raziskovanem območju apnenec in dolomit (Buser 2009). Preučene združbe uspevajo na kamniščih (LovrenčAK 1998, Vidic et al. 2015). Podnebje je hladno in vlažno, s povprečno letno višino padavin med $2500 \mathrm{~mm}$ in $3000 \mathrm{~mm}$ (ZuPANČIČ 1998) in srednjo letno temperature zraka med $-2{ }^{\circ} \mathrm{C}$ in $0{ }^{\circ} \mathrm{C}(\mathrm{CE}-$ GNAR 1998).

\subsection{Rezultati in razprava}

5.3.1 Pregled preučenih sintaksonov s tipi na novo opisanih združb

Thlaspietea rotundifolii Br.-Bl. 1948

Thlaspietalia rotundifolii Br.-Bl. in Br.-Bl. et Jenny 1926

Thlaspion rotundifolii Jenny-Lips 1930

Papaveri julici-Thlaspietum rotundifolii T. Wraber 1970

Crepidetum terglouensis Seibert 1977

Crepido terglouensis-Potentilletum nitidae ass. nov. hoc. loco, nomenklaturni tip, holotypus, je popis 12 $\mathrm{v}$ preglednici 1 .

-typicum, subass. nov., nomenklaturni tip je isti kot nomenklaturni tip asociacije

-caricetosum firmae, nomenklaturni tip, holotypus, je popis $5 \mathrm{v}$ preglednici 1 .

Saxifrago sedoidis-Geumetum reptantis nom. prov. Arabidetalia caeruleae Rübel ex Nordhagen 1937

Arabidion caeruleae Br.-Bl. In Br.-Bl. et Jenny 1926

Saxifrago carniolicae-Cerastietum uniflorae ass.

nov. hoc loco, nomenklaturni tip, holotypus, je popis $18 \mathrm{v}$ preglednici 1 .

Asplenietea trichomanis (Br.-Bl. in Meier et Br.-Bl. 1934) Oberd. 1977

Potentilletalia caulescentis Br.-Bl. in Br.-Bl. et Jenny 1926

Physoplexido comosae-Saxifragion petraeae Mucina et Theurillat 2015 (sin. Androsaco-Drabion tomentosae T. Wraber 1970, Phyteumato-Saxifragion petraeae Mucina in Šilc et Čarni 2012)

Potentilletum nitidae Wikus 1959

5.3.2 Vrsta Crepis terglouensis in združbe, v katerih uspeva v Sloveniji

Po delu Flora alpina (Aeschimann et al. 2004b: 668) je Crepis terglouensis vzhodnoalpska vrsta, značilnica zveze Thlaspion rotundifoliae, ki združuje rastje subal- 
pinsko-alpinskih melišč na karbonatni podlagi. Po njej se imenuje vzhodnoalpska alpinska združba Crepidetum terglouensis Seibert 1977, ki jo je s fitocenološko tabelo podrobno predstavil EgGENSBERGER (1994: 6466, 76-77). Nekaj podobnosti s to združbo naj bi imel tudi popis, ki ga je objavil SUTTER (1969: 353) in sta ga naredila skupaj s T. Wraberjem pod planinskim domom Planika pod Triglavom. T. Wraber (1972, 1990) triglavski dimek omenja predvsem v posebni obliki asociacije Papaveri julici-Thlaspietum rotundifolii. Našel ga je na dveh meliščih na Kredarici in na prelazu Grlo med Oltarjem in Dovškim križem. E. \& S. Pignatti $(2014,2016)$ to vrsto kot redko navajata $\mathrm{v}$ sestojih dveh drugih alpinskih meliščnih združb Leontodontetum montani in Papaveretum rhaetici in v posebni obliki (Seleginella-Homogyne) asociacije Seslerio-Caricetum sempervirentis. Dve novi nahajališči te vrste v slovenskih Alpah (na grušču pod Prestreljenikom v Kaninskem pogorju - PrAProtniK, 1997, 2002 in na melišču pod Stolom v Karavnkah - NovAK 2015) fitocenološko do zdaj nista popisani. Na nahajališču pod Stolom, Novak (ibid.) omenja spremljevalni vrsti Sesleria caerulea in Campanula cochleariifolia. Pri naših raziskavah smo večino popisov triglavskega dimka naredili na drobnem grušču na Glavi nad Zaplanjo pod Triglavom in na sosednjih vzpetinah $\mathrm{v}$ smeri proti Doliču (9648/2) - slika 1, novo nahajališče pa smo našli tudi na enakem rastišču na prelazu Vrata med Zelnarico in Zadnjim Voglom (9648/4), na nadmorski višini 2192 m (leg. \& det. I. Dakskobler, B. Anderle in B. Zupan, 23. 8. 2016, herbarij LJS), kar je novo nahajališče te vrste $\mathrm{v}$ novem kvadrantu (slika 2). Po primerjavi naših popisov z vrstama Crepis terglouensis in/ali Cerastium uniflorum s podobnimi popisi $\mathrm{T}$. WraberJa (1972), so se posebej združevali popisi, v katerih skupaj prevladujeta vrsti Potentilla nitida in Crepis terglouensis (slika 3). Očitno smo popisali prehodno obliko alpinskega rastja med meliščnimi združbami, združbami skalnih razpok in kamnitih travišč, ki jo je z enim popisom ponazoril že SutTer (ibid.) in jo omenja tudi EgGeNSBERgER (ibid.). Zato smo izdelali sintezno tabelo (Dodatek 1), v kateri smo 15 popisov preučenih sestojev primerjali s 33 popisi asociacije $\mathrm{Po}$ tentilletum nitidae (T. Wraber 1972, preglednica 3) in s 25 popisi asociacije Crepidetum terglouensis (EGGENsBERGER 1994, preglednica 6, stolpci 85-111). Rezultati (slika 4) kažejo na večjo podobnost naših popisov s sestoji asociacije Potentilletum nitidae. Mogoče bi jih bilo torej uvrstiti v novo subasociacijo, Potentilletum nitidae crepidetosum terglouensis, toda analiza diagnostičnih vrst (preglednica 2, stolpec 1) kaže na prevlado meliščnih vrst iz reda Thlaspietelia rotundifolii $(37,43$ \%) nad diagnostičnimi vrstami skalnih razpok iz reda
Potentilletalia caulescentis (23,8 \%). Večino popisov smo naredili na drobnem grušču, med katerim je skoraj vedno nekaj dobro razkrojenega humusa (sprstenine), na uravnavah, kjer se razmeroma dolgo zadržuje sneg. Dominantna vrsta popisanih sestojev je navadno Potentilla nitida, njeno družno uspevanje s tipično meliščno vrsto Crepis terglouensis na razmeroma majhnih, a ekološko svojskih površinah med skalovjem (droben grušč) lahko označuje določeno razvojno (sukcesijsko) stopnjo, ki je morda deloma povezana tudi z zdajšnjimi podnebnimi spremembami, očitno manjšo količino snežnih padavin in krajšimi obdobji s snežno odejo na planotah zahodno od Triglava, kar dokazujejo meritve bližnjega Triglavskega ledenika na podobni nadmorski višini (GABrovec et al. 2014). To sukcesijsko stopnjo lahko obravnavamo tudi v rangu nove asociacije Crepido terglouensis-Potentilletum nitidae, ki jo uvrščamo v zvezo Thlaspion rotundifolii. Diagnostične vrste nove asociacije so Potentilla nitida, Crepis terglouensis, Eritrichium nanum in Minuartia cherlerioides. Razlikujemo dve subasociaciji, tipično (-typicum) in -caricetosum firmae. Razlikovalnice slednje so vrste Carex firma, Silene acaulis in Salix retusa, ki morda nakazujejo prehod proti kamnitim alpinskim traviščem iz asociacije Gentiano terglouensis-Caricetum firmae.

5.3.3 Vrsta Cerastium uniflorum in združbe, v katerih uspeva v Sloveniji

Cerastium uniflorum je alpsko-karpatska vrsta, značilnica zveze silikatnih melišč subalpinskega in alpinskega pasu Androsacion alpinae (Aeschimann et al. 2004a: 298). Njeno razširjenost v Sloveniji prikazuje slika 5. Znana nahajališča so le na Triglavu in v njegova soseščini, na Rjavini in Mangartu. T. Wraber (1990: 130, 1972) navaja njeno pojavljanje v sestojih asociacij Potentilletum nitidae in Papaveri julici-Thlaspietum rotundifolii. E. \& S. Pignatti $(2014,2016)$ sta to vrsto popisala v sestojih asociacij Drabetum hoppeanae, Saxifragetum sedoidis, Leontodontetum montani, Papaveretum rhaetici, Festucetum pulchellae, Vitaliano-Eritrichetum nani, Arabidetum caeruleae, Saxifragetum bryoidis in $\mathrm{v}$ še nekaterih drugih. $\mathrm{V}$ naših popisih jo imamo kot posamično primes $\mathrm{v}$ sestojih asociacij Saxifrago paniculatae-Caricetum fuliginosae (DАкsкоBLER \& Surina 2017) in Crepido-Potentilletum nitidae (ta članek), je pa ena od prevladujočih vrst $\mathrm{v}$ šestih popisih na večinoma drobnem grušču in na krajih, kjer se dolgo zadržuje sneg (slika 6, popisi 17-21 v preglednici 1). Ti popisi so se združevali ločeno od drugih popisov alpinskih melišč in alpskih trat na skalnatih policah (slika 2). Poleg vrste Cerastium uniflorum je na njih 
najbolj pogosta vrsta Saxifrage sedoides, zato smo jih primerjali s popisi asociacije Saxifragetum sedoidis iz Dolomitov (E. \& S. PignatTi 2016: 204: asociacijska preglednica 9.2, 406-407) - Dodatek 2. V Krnskem pogorju je SuRina (2005) s fitocenološko tabelo predstavil nekoliko podoben sintakson Saxifragetum stellaro-sedoidis var. geogr. Ranunculus traunfellneri, vendar v njegovih sestojih ni popisal enocvetne smiljke in tudi v izvornem opisu te asociacije (ENGLISCH 1999) je ni. Floristična podobnost naših popisov s popisi sestojev asociacije Saxifragetum sedoidis je precej manj kot polovična, le 41-odstotna (SøRENSEN 1948), kar ne dopušča uvrstitve v to asociacijo. Tudi te sestoje lahko razložimo kot razvojno (sukcesijsko) stopnjo, saj vrsta Cerastium uniflorum $\mathrm{v}$ skrajnih razmerah alpinskega pasu lahko uspeva na različnih rastiščih in porašča tudi površine, ki jih morda pred 50 ali 100 leti še ni poraščala. Zato in na podlagi sestave diagnostičnih vrst (tabela 2, stolpec 2) jih uvrščamo v novo asociacijo Saxifrago carniolicae-Cericetum uniflorae in v zvezo Arabidion caeruleae. Njene diagnostične vrste so Cerastium uniflorum, Saxifraga sedoides in Saxifraga exarata subsp. carniolica. Slednja je endemit Jugovzhodnih Alp in značilnica alpinskih združb skalnih razpok (Potentilletum nitidae, Potentillo clusianae-Campanuletum zoysii) - T. Wraber (2006: 70) in kamnitih travišč iz zvez Caricion firmae in Seslerion variae (Aeschimann et al. 2004: 2010). Prvi dve našteti vrsti označujeta alpinsko združbo drobnega grušča v območjih $\mathrm{z}$ dolgotrajno snežno odejo, tretja pa novo asociacijo označuje predvsem fitogeografsko in deloma kaže na razvojno povezanost združb drobnega grušča z združbami kamnitih alpskih trat in skalnih razpok. V enem sestoju je v veliki količini prisotna vrsta Sagina saginoides, ki kaže na krajevno boljše talne razmere, $\mathrm{z}$ obilico drobne preperine (sprstenine).

\subsubsection{Vrsta Geum reptans v Triglavskem pogorju}

Tudi južnoevropsko montansko vrsto Geum reptans Aeschimann et al. (2004a: 758) uvrščajo med značilnice zveze Androsacion alpinae. V Julijskih Alpah so bila do zdaj znana le nahajališča na Mangartu in Kanjavcu (T. Wraber 2006, Zupan \& DAKSKObler 2007, slika 7). 8. 8. 2016 smo našli novo nahajališče te meliščne vrste na zahodnih pobočjih Triglava, v krnici pod Vrhom Zelenic, na nadmorski višini 2060 m (DAкsKoBLER 2017). Rastišče je ustaljeno melišče z različno velikimi kosi kamenja in precej poraslo. Njegova vrstna sestava je $\mathrm{v}$ stolpcu 2, v preglednici 3. V stolpcu 1 te tabele je popis združbe na nahajališču na Temenu med Kanjavcem in Poprovcem (ZupAN \& DAKsKobler 2007), ki smo ga označili kot prehod med združbo triglavske rože (Potentilletum nitidae) in sestojem asociacije Papaveri julici-Thlaspietum rotundifolii. Sestoja pod Vrhom Zelenic ne moremo uvrstiti v nobeno od obeh navedenih asociacij. Označuje združbo melišč $s$ precej vrstami kamnitih alpskih trat (iz asociacije Gentiano terglouensis-Caricetum firmae). Začasno jo uvrščamo v provizorno asociacijo Saxifrago sedoidis-Geumetum reptantis in $\mathrm{v}$ zvezo Thlaspion rotundifolii. Združbo poleg prevladujoče vrste Geum reptans označujejo predvsem vrste iz rodu Saxifraga (S. sedoides, $S$. paniculata, S. aizoides, S. crustata, S. squarrosa) ter dve tipični meliščni vrsti: Festuca nitida in Poa minor.

\subsection{Sklepne misli}

Razultat florističnega kartiranja v zahodnem delu Triglava (Glava v Zaplanji, Vrh Zelenic) in na prevalu Vrata med Veliko Zelnarico in Zadnjim Voglom so nova nahajališča treh v Sloveniji redkih vrst alpinskih melišč in/ali skalnih razpok, ki so uvrščene na rdeči seznam (Anon. 2002): Cerastium uniflorum (nova nahajališča v kvadrantu 9648/2), Crepis terglouensis (nova nahajališča v kvadrantih 9648/2 in 9648/4) ter Geum reptans (novo nahajališče v že znanem kvadrantu, 9648/2, novost v flori Triglava). Fitocenološko smo popisali združbe, v katerih uspevajo navedene vrste in ugotovili njihove netipične sestoje na drobnem grušču z drobno preperino (sprstenino), ki so prehodni med alpinskimi združbami skalnih razpok, melišč in snežnih dolinic ter so lahko sukcesijska stopnja na površinah, ki so bile pred desetletji precej dlje pokrita s snegom. Na podlagi primerjave s podobnimi združbami iz Vzhodnih in Jugovzhodnih Alp smo opisali dve novi asociaciji Crepido terglouensis-Potentilletum nitidae in Saxifrago carniolicae-Cerastietum uniflorae. Čeprav so njuni sestoji v bližini precej obiskanih planinskih poti, ki vodijo proti Triglavu, jih vsako leto večji poletni turistični obisk za zdaj ne ogroža, saj drugih posegov v ta prostor tu ni in sta vrsti Cerastium uniflorum in Crepis terglouensis precej neopazni, še posebej v času, ko ne cvetita. Novo nahajališče vrste Geum reptans, ki tudi raste v netipični meliščni združbi (Saxifrago sedoidis-Geumetum reptantis nom. prov.), je v odmaknjenem brezpotju v krnici pod Vrhom Zelenic in nanj za zdaj človek nima nobenega neposrednega vpliva. 


\section{ACKNOWLEDGEMENTS}

We are extremely grateful to Prof. Dr. Andrej Martinčič for his determination of mosses. Iztok Sajko prepared Figure 1 for print. Academician Dr. Mitja Zupančič and Prof. Nanika Holz helped us with valuable im- provements and corrections. The authors acknowledge the financial support from the Slovenian Research Agency (research core funding No. P1-0236). English translation by Andreja Šalamon Verbič.

\section{REFERENCES - LITERATURA}

Aeschimann, D., K. Lauber, D. M. Moser \& J.-P. Theurillat, 2004a: Flora alpina. Bd. 1: Lycopodiaceae-Apiaceae. Haupt Verlag, Bern, Stuttgart, Wien.

Aeschimann, D., K. Lauber, D. M. Moser \& J.-P.Theurillat, 2004b: Flora alpina. Bd. 2: Gentianaceae-Orchidaceae. Haupt Verlag, Bern, Stuttgart, Wien.

Aeschimann, D., K. Lauber, D. M. Moser \& J.-P.Theurillat, 2004c: Flora alpina. Bd. 3: Register. Haupt Verlag, Bern, Stuttgart, Wien.

Anonymous, 2002: Pravilnik o uvrstitvi ogroženih rastlinskih in živalskih vrst v rdeči seznam. Uradni list RS $82 / 2002$.

Braun-Blanquet, J., 1964: Pflanzensoziologie. Grundzüge der Vegetationskunde. 3. Auflage. Springer, Wien New York.

Buser, S., 2009: Geološka karta Slovenije 1: 250.000. Geological map of Slovenia 1: 250,000. Geološki zavod Slovenije, Ljubljana.

Cegnar, T., 1998: Temperatura zraka. In: J. Fridl, D. Kladnik, M. Orožen Adamič \& D. Perko (eds.): Geografski atlas Slovenije. Država v prostoru in času. Državna založba Slovenije, Ljubljana, pp. 100-101.

Daksковler, I., 2017: Dve botanični opombi pod črto: plazeča sretena (Geum reptans) pod Triglavom in venerini laski (Adiantum capillus-veneris) v grapi Volarje pri Seliščih. Proteus (Ljubljana) 79 (5): 216-223.

DAKsкobler, I. \& B. SURINA, 2017: Phytosociological analysis of alpine swards and heathlands (pioneer patches) on ridges and peaks in the Julian Alps (NW Slovenia). Hacquetia (Ljubljana) 16 (1): 49-171. https://doi.org/10.1515/ hacq-2016-0022

Eggensberger, P., 1994: Die Pflanzengesellschaften der subalpinen umd alpinen Stufe der Ammergauer Alpen und ihre Stellung in den Ostalpen. Ber. Bayer. Bot. Ges., Beihefte 8: 3-239.

Englisch, T., 1999: Multivariate Analysen zur Synsystematik und Standortsökologie der Schneebodenvegetation (Arabidetalia caerulae) in den Nördlichen Kalkalpen. Stapfia (Linz) 59: 1- 211 + Tables.

Englisch, T., M. Valachovič, L. Mucina, G. Grabherr, G.\& T. Ellamuer, 1993: Thlaspietea rotundifolii. In: G. Grabherr \& L. Mucina (eds.): Die Pflanzengesellschaften Österreichs. Teil II: Natürliche waldfreie Vegetation, Gustav Fischer Verlag, Jena - Stuttgart - New York, pp. 276-342.

Gabrovec, M., M. Hrvatin, B. Komac, J. Ortar, M. Pavšek, M. Topole, M. Triglav Čekada \& M. Zorn, 2014: Triglavski ledenik. Založba ZRC, Ljubljana.

LovrenčAK, F., 1998: Prsti. In: J. Fridl, D. Kladnik, M. Orožen Adamič \& D. Perko (eds.): Geografski atlas Slovenije. Država v prostoru in času. Državna založba Slovenije, Ljubljana, pp. 114-115.

MAAREL van der, E., 1979: Transformation of cover-abundance values in phytosociology and its effects on community similarity. Vegetatio 39 (2): 97-114. https://doi.org/10.1007/BF00052021

Martinčič, A., T. Wraber, N. Jogan, A. Podobnik, B. Turk, B. Vreš, V. Ravnik, B. Frajman, S. Strgulc Krajšek, B. TrČak, T. Bačič, M. A. Fischer, K. Eler \& B. Surina, 2007: Mala flora Slovenije. Ključ za določanje praprotnic in semenk. Četrta, dopolnjena in spremenjena izdaja. Tehniška založba Slovenije, Ljubljana.

Mucina, L., H. Bültmann, K. Dierssen, J-P. Theurillat, T. Raus, A. Čarni, K. Šumberová, W. Willner, J. Dengler, R. Gavilán García, M. Chytrí, M. Hájek, R. Di Pietro, D. Iakushenko, J. Pallas, F. J. A. Daniëls, E., Bergmeier, A. Santos Guerra, N. Ermakov, M. Valachovič, J. H. J. Schaminée, T. Lysenko, Y. P. Didukh, S. Pignatti, J. S. Rodwell, J. Capelo, H. E. Weber, A. Solomeshch, P. Dimopoulos, C. Aguiar, S. M. Hennekens \& L. TichÝ, 2016: Vegetation of Europe: hierarchical floristic classification system of vascular plant, bryophyte, lichen, and algal communities. Applied Vegetation Science 19, Suplement 1: 3-264. http://dx.doi.org/10.1111/avsc.12257 
NovaK, Š., 2015: Crepis terglouensis (Hacquet) Kerner. Notulae ad floram Sloveniae. Hladnikia (Ljubljana) 36: 47-50.

Pignatti, E. \& S. Pignatti, 2014: Plant Life of the Dolomites. Vegetation Structure and Ecology. Publication of the Museum of Nature South Tyrol Nr. 8, Naturmuseum Südtirol, Bozen, Springer Verlag, Heidelberg. https://doi. org/10.1007/978-3-642-31043-0

Pignatti, E. \& S. Pignatti, 2016: Plant Life of the Dolomites.Vegetation Tables. Publication of the Museum of Nature South Tyrol Nr. 11, Bozen, Springer Verlag, Heidelberg. https://doi.org/10.1007/978-3-662-48032-8

PoDANI, J., 2001: SYN-TAX 2000. Computer Programs for Data Analysis in Ecology and Systematics. User's Manual, Budapest.

Praprotnik, N., 1997: Crepis terglouensis (Hacq.) A. Kerner. In: Jogan N.: Nova nahajališča. Hladnikia (Ljubljana) 8/9: 59.

Praprotnik, N., 2002: Triglavski dimek. Gea (Ljubljana) 12 (5): 65-66.

Seliškar, T., B. Vreš \& A. SelišKar, 2003: FloVegSi 2.0. Fauna, Flora, Vegetation and Paleovegetation of Slovenia. Computer programme for arranging and analysis of biological data. Biološki inštitut ZRC SAZU, Ljubljana.

SørENSEN, Th., 1948: A method of establishing groups of equal amplitude in plant sociology based on similarity of species content. Det Kongelige Danske Videnskaberns Selskab, Biologiske Skrifter (København) 5 (4): 1-34.

SurINA, B., 2005: Subalpinska in alpinska vegetacija Krnskega pogorja v Julijskih Alpah. Scopolia (Ljubljana) 57: $1-122$.

Sutter, R., 1969: Ein Beitrag zur Kenntnis der soziologischen Bindung süd-südostalpiner Reliktendemismen. Acta Bot. Croatica (Zagreb) 28: 349-365.

ŠILC, U. \& A. ČArNI, 2012: Conspectus of vegetation syntaxa in Slovenia. Hacquetia (Ljubljana) 11 (1): 113-164. https://doi.org/10.2478/v10028-012-0006-1

Theurillat, J.-P., 2004: Pflanzensoziologisches System. In: Aeschimann, D., K. Lauber, D. M. Moser \& J.-P. Theurillat: Flora alpina 3: Register. Haupt Verlag, Bern, Stuttgart, Wien, pp. 301-313.

Vidic, N. J., T. Prus, H. GrČman, M. Zupan, A. Lisec, T. Kralj, B, Vrščaj, J. Rupreht, M. Šporar, M. SuhaDolc, R. Mihelič, R. \& F. Lobnik, 2015: Tla Slovenije s pedološko karto v merilu 1: 250 000. Soils of Slovenia with soil map 1: 250 000. European Union \& University of Ljubljana, Luxemburg, Ljubljana.

Wraber, T., 1972: Contributo alla conoscenza della vegetazione pioniere (Asplenietea rupestria e Thlaspeetea rotundifolii) delle Alpi Giulie. Tesi di laurea. Università degli Studi di Trieste, Facoltà di Scienze, Trieste (Doktorska naloga, $81 \mathrm{pp}$.)

Wraber, T., 1990: Sto znamenitih rastlin na Slovenskem. Prešernova družba, Ljubljana.

Wraber, T., 2006: 2 Sto alpskih rastlin na Slovenskem. Prešernova družba, Ljubljana.

Zupan, B. \& I. Daksкobler, 2007: Geum reptans L. Notulae ad floram Sloveniae 83. Hladnikia (Ljubljana) 20: 36-38.

Zupančıč, B., 1998: Padavine. In: J. Fridl, D., Kladnik, M. Orožen Adamič \& D. Perko (eds.): Geografski atlas Slovenije. Država v prostoru in času. Državna založba Slovenije, Ljubljana, pp. 98-99.

Figures 7-14: Photo/Foto: I. Dakskobler 


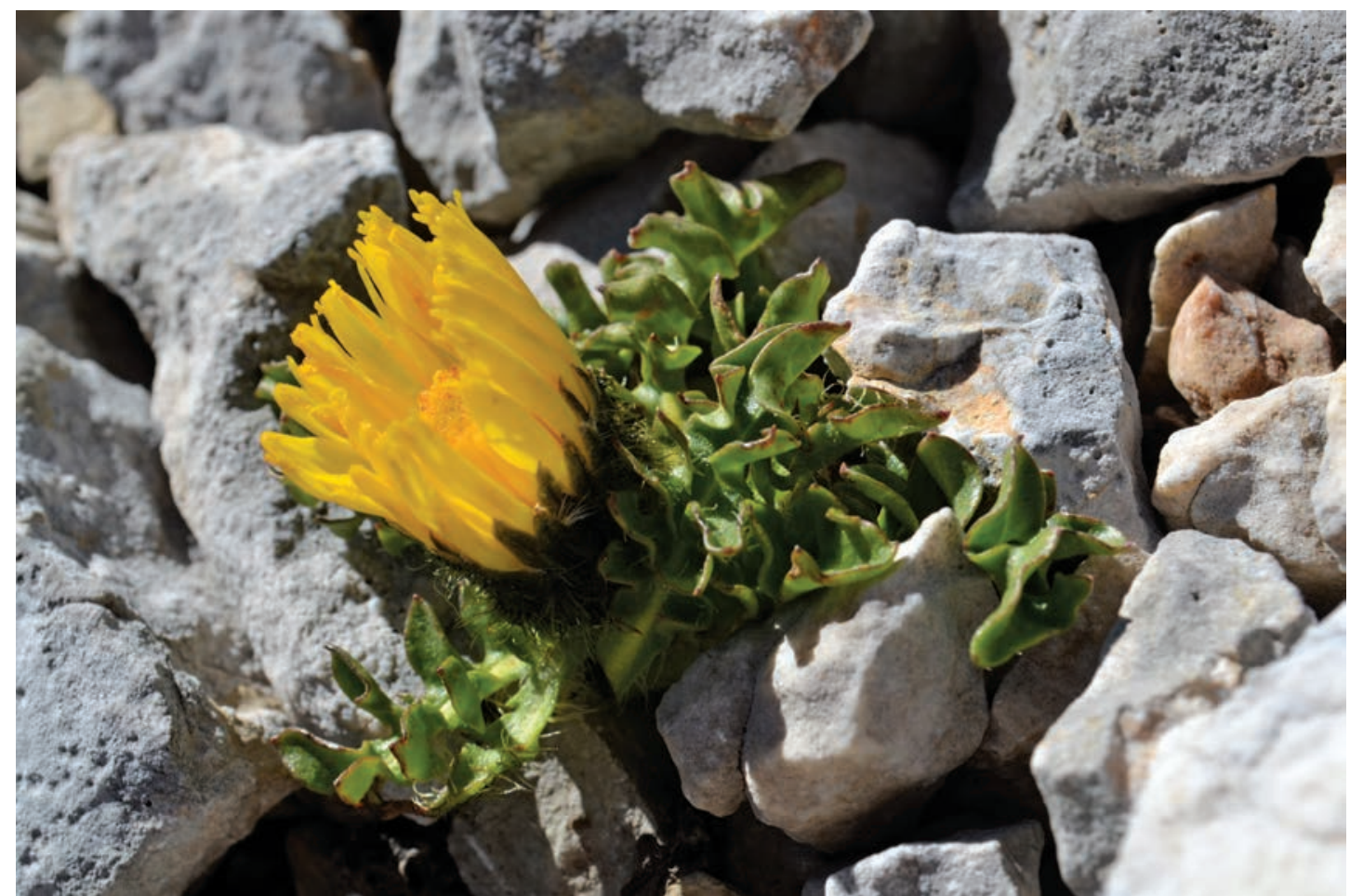

Figure 7: Crepis terglouensis

Slika 7: Triglavski dimek (Crepis terglouensis)

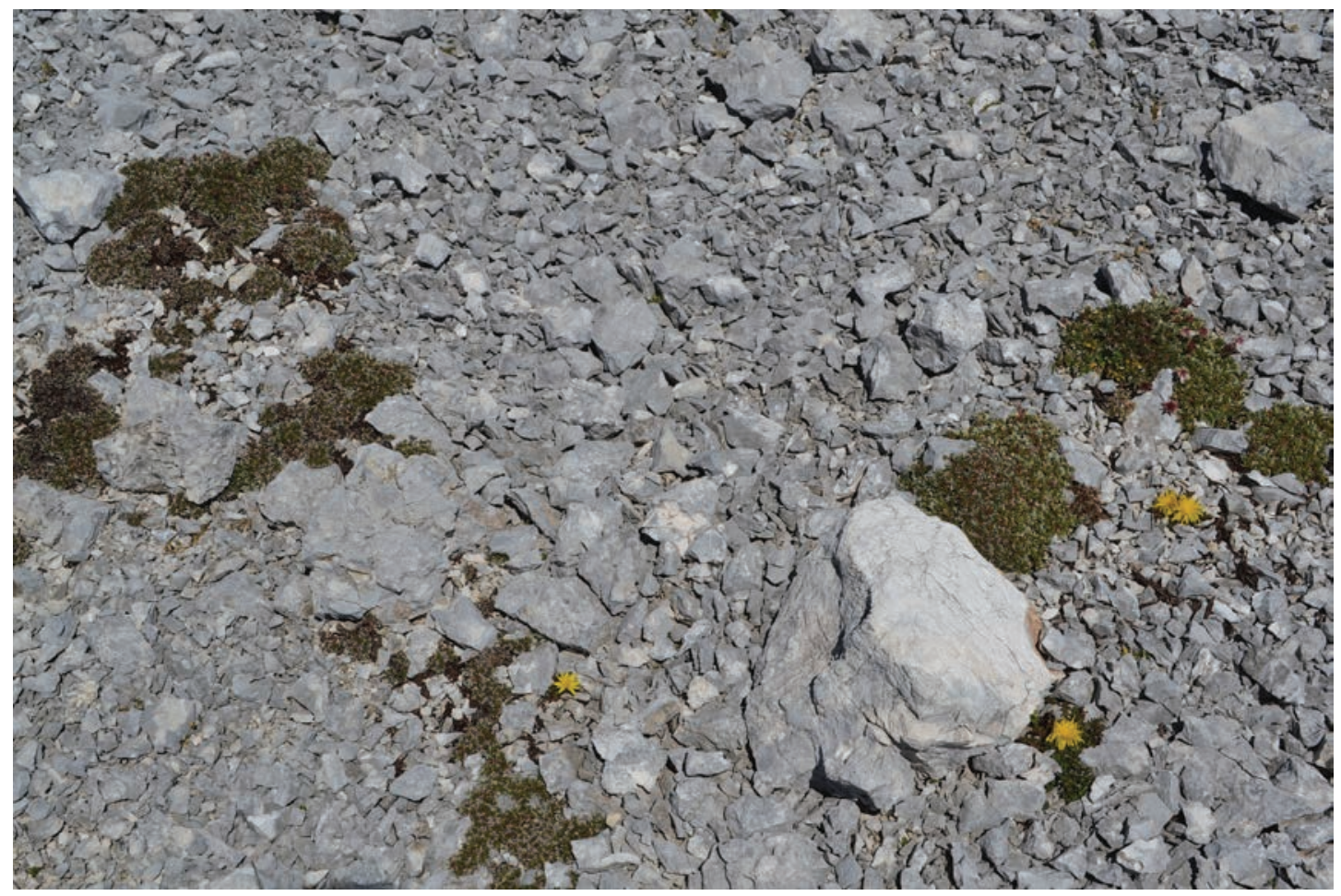

Figure 8: Stand of the association Crepido terglouensis-Potentilletum nitidae Slika 8: Sestoj asociacije Crepido terglouensis-Potentilletum nitidae 


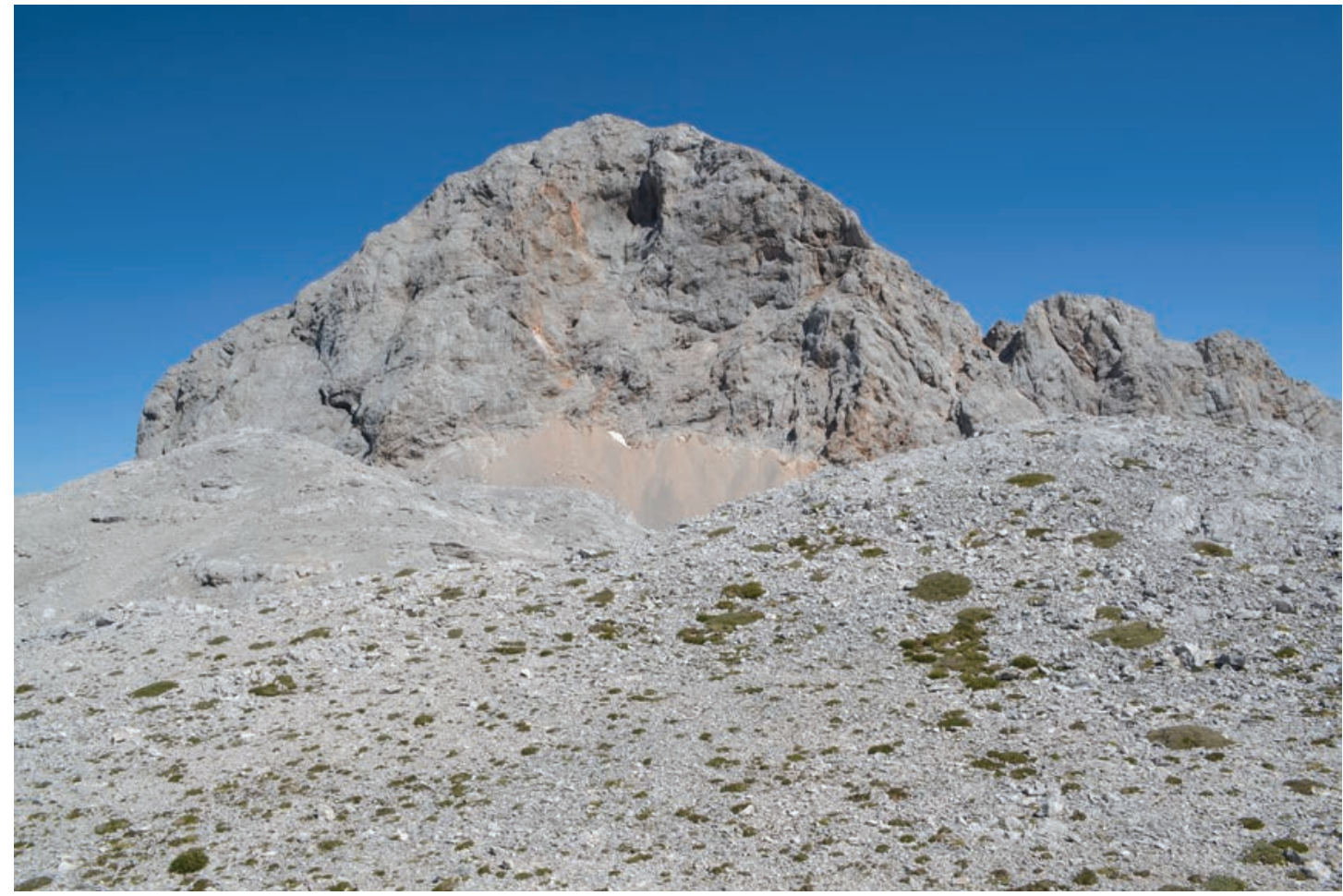

Figure 9: Glava nad Zaplanjo under Mt. Triglav, the area where we have recorded stands of the association Crepido terglouensis-Potentilletum nitidae

Figure 9: Glava nad Zaplanjo, območje, kjer smo popisovali sestoje asociacije Crepido terglouensis-Potentilletum nitidae

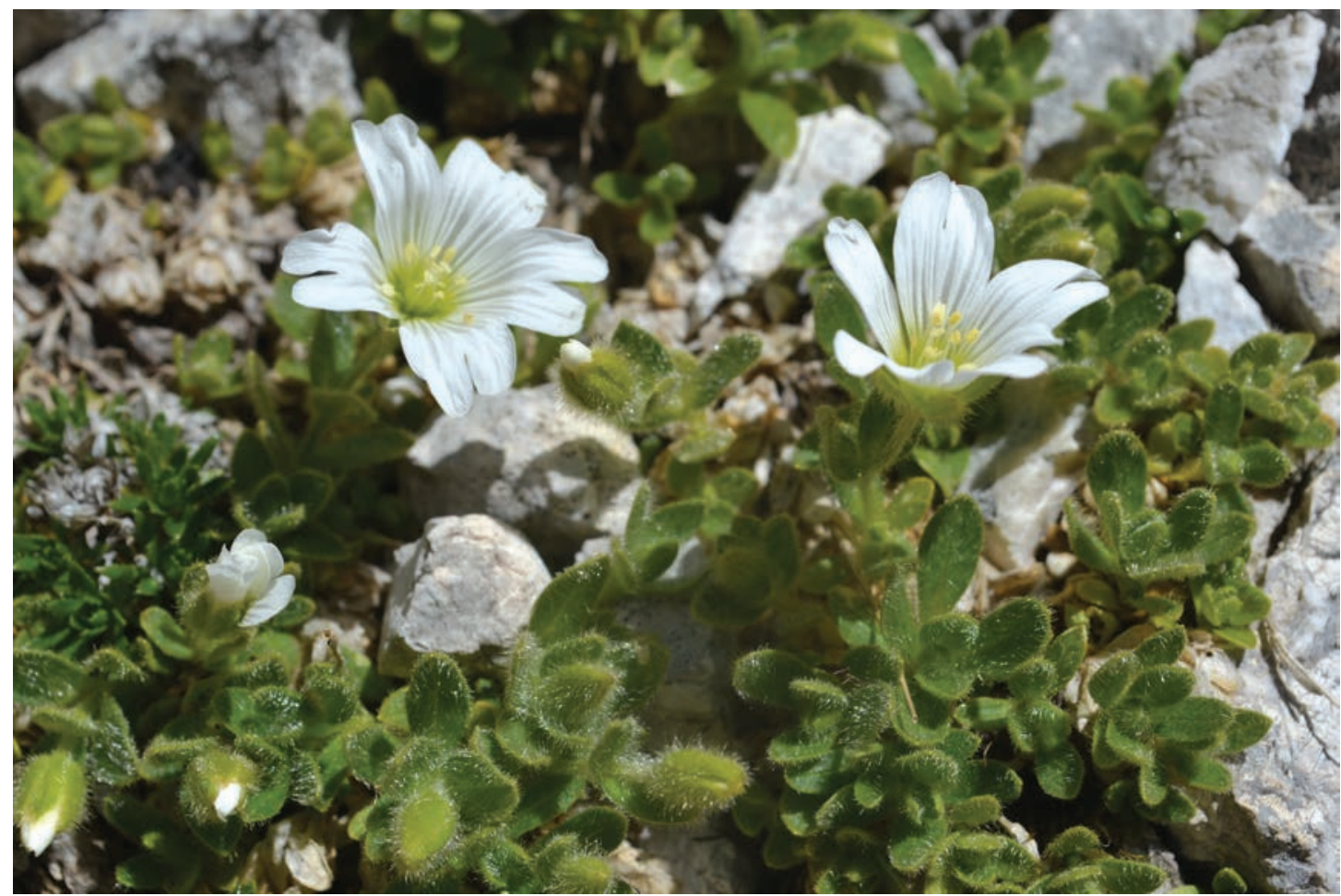

Figure 10: Cerastium uniflorum

Slika 10: Enocvetna smiljka (Cerastium uniflorum) 


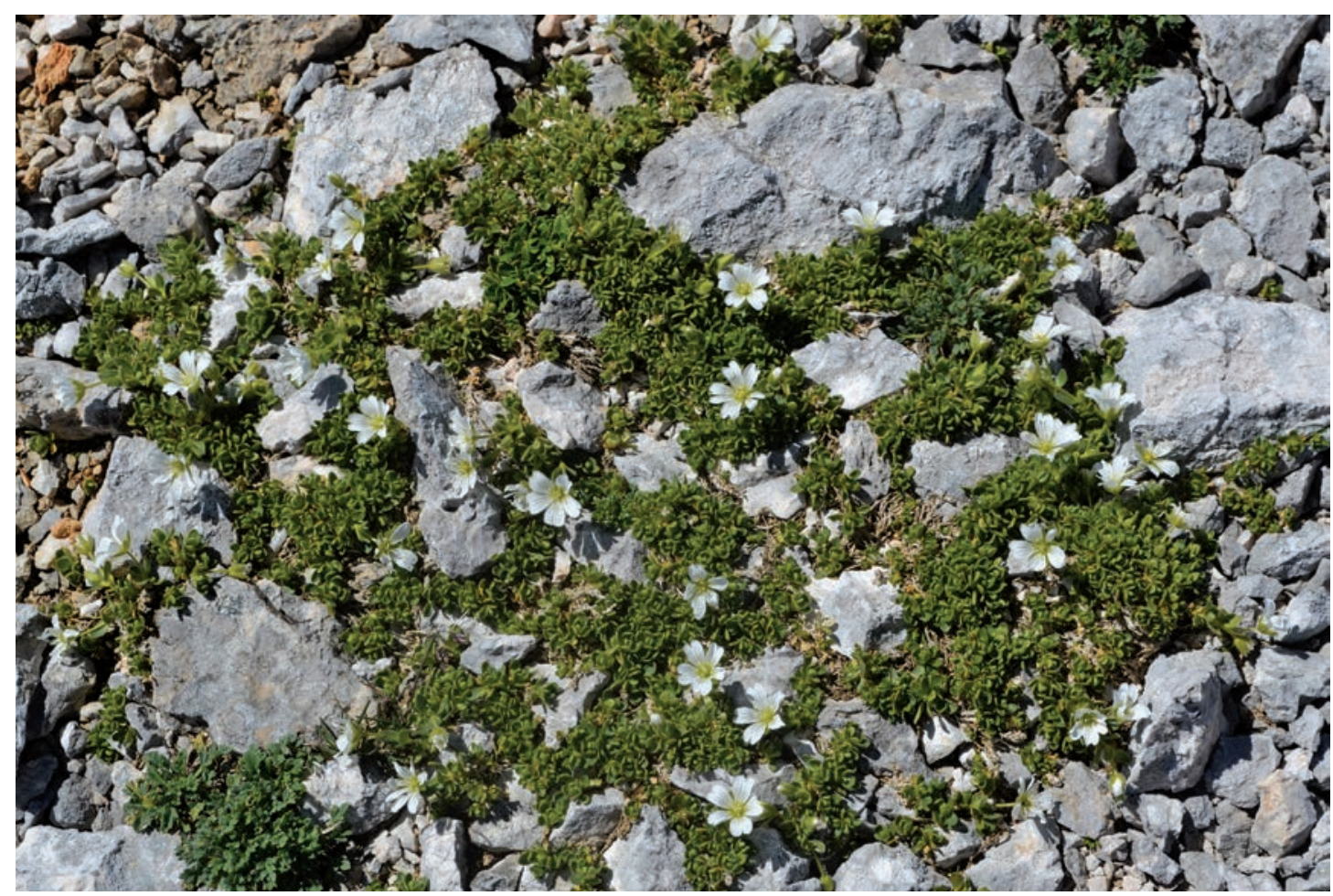

Figure 11: Stand of the association Saxifrago carniolicae-Cerastietum uniflorae Slika 11: Sestoj asociacije Saxifrago carniolicae-Cerastietum uniflorae

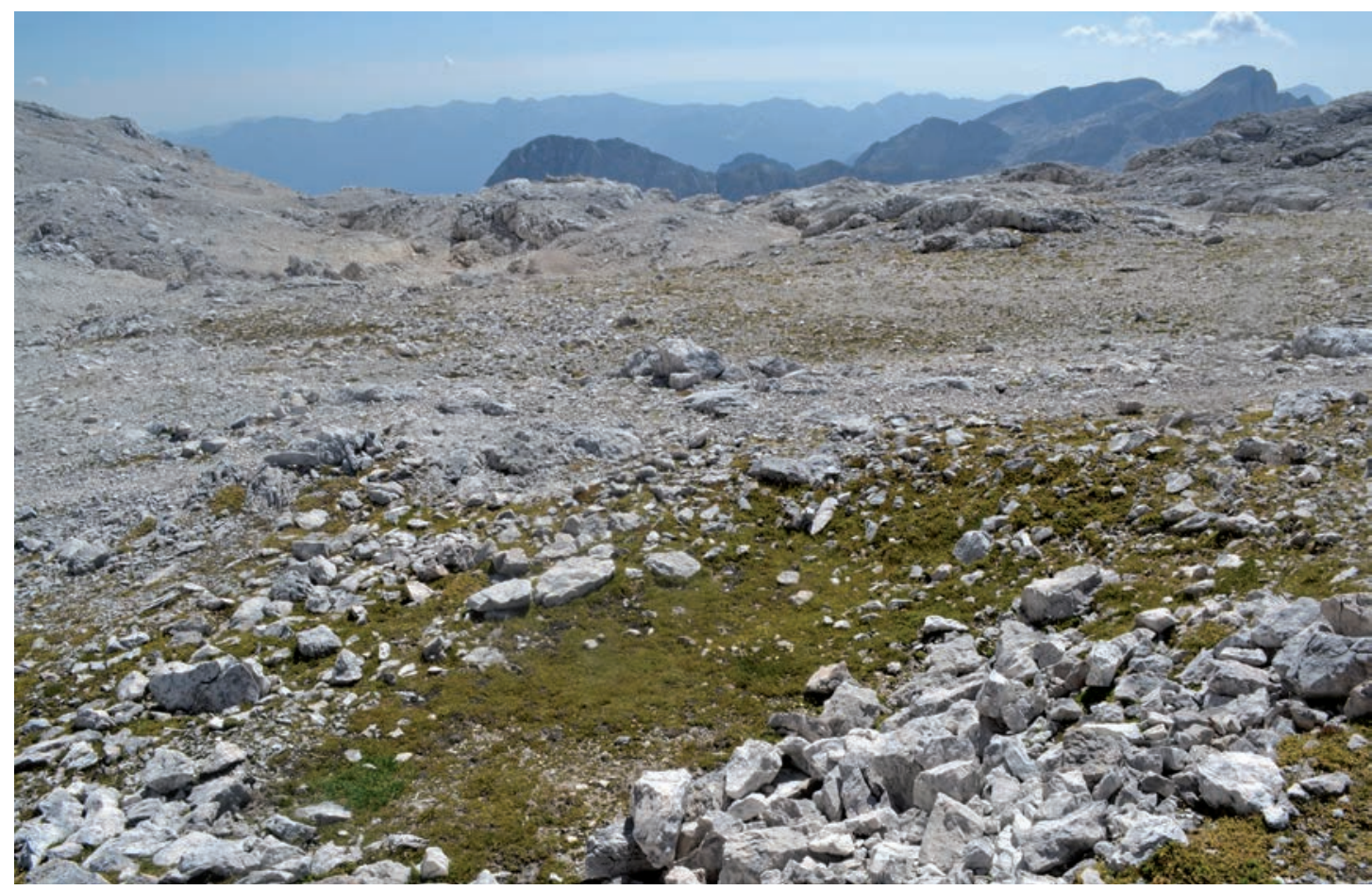

Figure 12: Plateau west from Mt. Triglav, area, where we have recorded stands of the association Saxifrago carniolicaeCerastietum uniflorae

Slika 12: Planota zahodno od Triglava, kjer smo popisovali sestoje asociacije Saxifrago carniolicae-Cerastietum uniflorae 


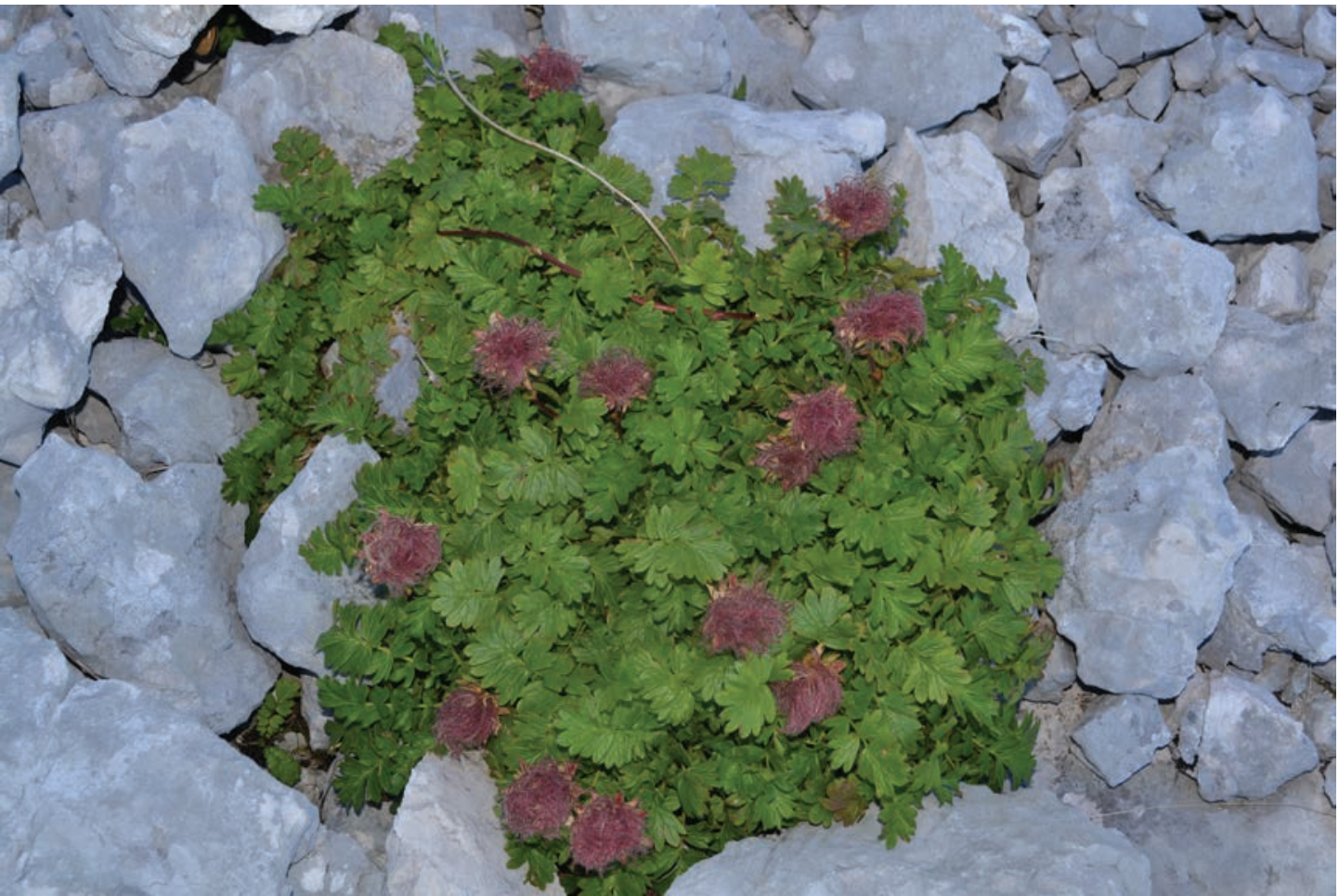

Figure 13: Geum reptans under Mt. Triglav

Slika 13: Plazeča sretena (Geum reptans) pod Triglavom

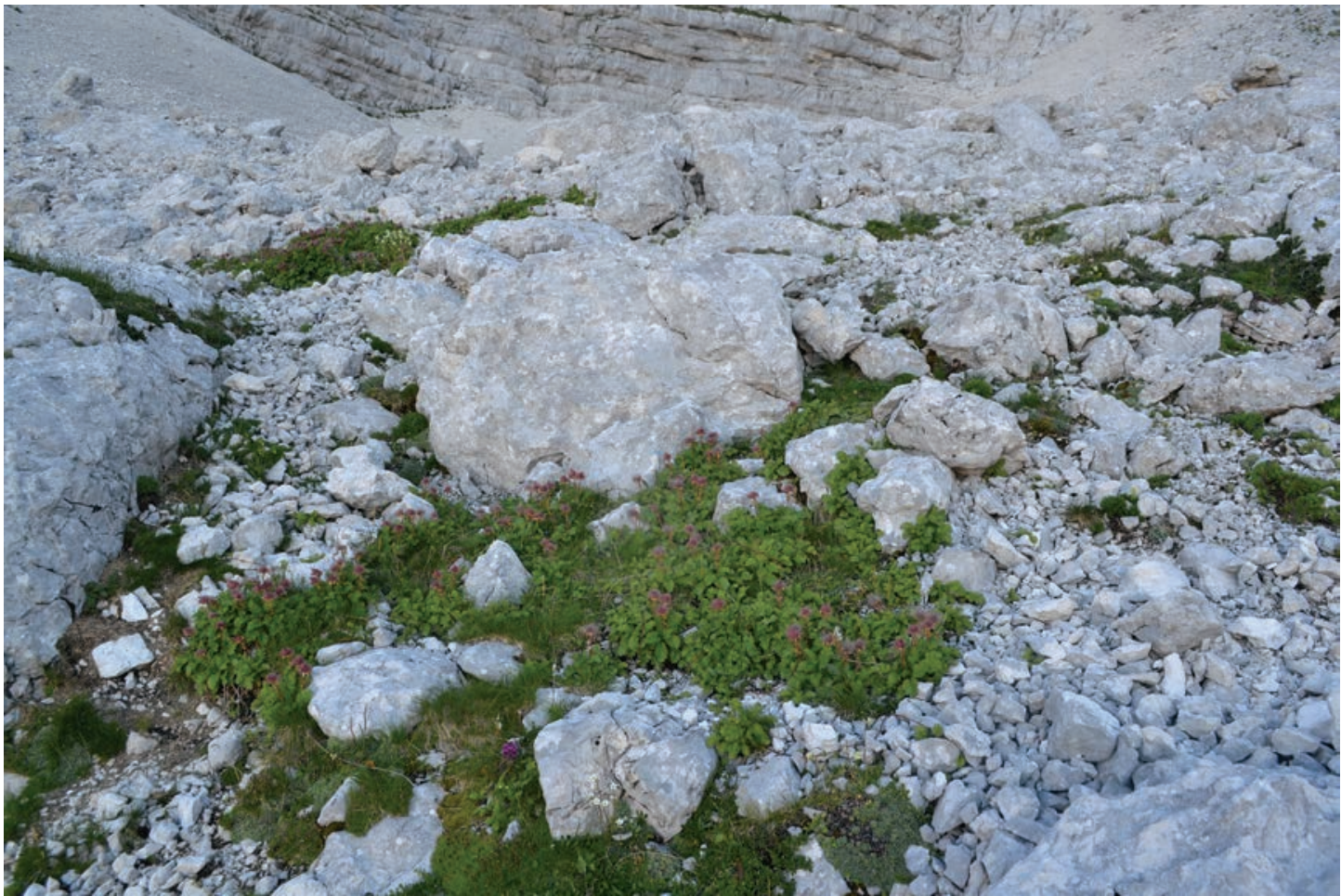

Figure 14: Stand of the association Saxifrago sedoidis-Geumetum raptantis Slika 14: Sestoj asociacije Saxifrago sedoidis-Geumetum raptantis 
Table 1 : Crepido terglouensis-Potentilletum nitidae and Saxifrago carniolicae-Cerastietum uniflorae Preglednica 1: Crepido terglouensis-Potentilletum nitidae in Saxifrago carniolicae-Cerastietum uniflorae

Number of relevé

(Zaporedna številka popisa)

Database number of relevé

(Delovna številka popisa)

Author of the relevé

(Avtor popisa)

Elevation in $\mathrm{m}$ (Nadmorska višina $\mathrm{v} \mathrm{m}$ )

Aspect (Lega)

Slope in degree

(Nagib v stopinjah)

Parent material

(Matična podlaga)

Soil (Tla)

Stoniness in \% (Kamnitost v \%)

Cover of herb layer in \%

(Zastiranje zeliščne plasti $\mathrm{v} \%$ )

Cover of moss layer in \%

(Zastiranje mahovne plasti v \%)

Number of species (Število vrst)

Relevé area

(Velikost popisne ploskve)

Date of taking relevé

(Datum popisa)

Locality (Nahajališče)

Coordinate GK Y (D-48)

Coordinate GK X (D-48) $\begin{array}{lllllllllllllllllllll}1 & 2 & 3 & 4 & 5 & 6 & 7 & 8 & 9 & 10 & 11 & 12 & 13 & 14 & 15 & 16 & 17 & 18 & 19 & 20 & 21\end{array}$

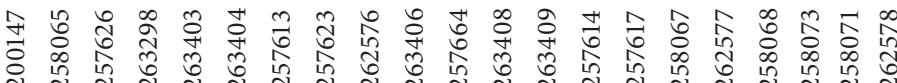

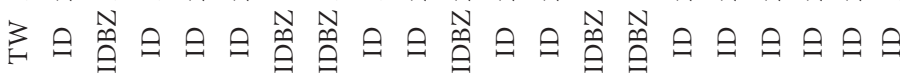

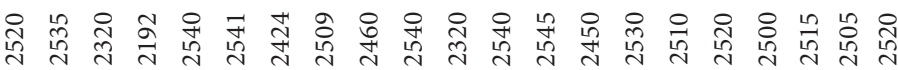

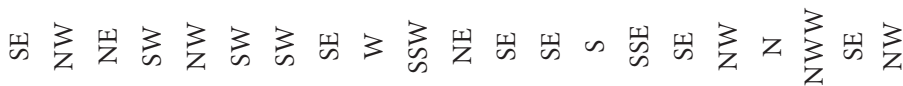

$\begin{array}{lllllllllllllllllllll}10 & 3 & 5 & 0-3 & 10 & 5 & 5 & 2 & 30 & 5 & 3 & 10 & 5 & 15 & 15 & 1 & 20 & 25 & 35 & 5 & 20\end{array}$

Gr Gr Gr Gr Gr Gr Gr Gr Gr Gr Gr Gr Gr Gr Gr Gr Gr Gr Gr Gr Gr $\mathrm{Li} \quad \mathrm{Li} \quad \mathrm{Li} \quad \mathrm{Li} \quad \mathrm{Li} \quad \mathrm{Li} \quad \mathrm{Li} \quad \mathrm{Li} \quad \mathrm{Li} \quad \mathrm{Li} \quad \mathrm{Li} \quad \mathrm{Li} \quad \mathrm{Li} \quad \mathrm{Li} \quad \mathrm{Li} \quad \mathrm{Re} \quad \mathrm{Li} \quad \mathrm{Li} \quad \mathrm{Li} \quad \mathrm{Li} \quad \mathrm{Li}$

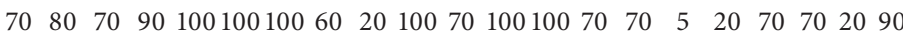
E1 $30 \begin{array}{llllllllllllllllllll}30 & 30 & 30 & 40 & 40 & 30 & 40 & 80 & 30 & 30 & 30 & 40 & 30 & 30 & 90 & 90 & 30 & 30 & 80 & 30\end{array}$ E0

$\begin{array}{lllllllllllllllllllll}18 & 14 & 10 & 12 & 19 & 15 & 7 & 3 & 5 & 6 & 3 & 12 & 18 & 15 & 11 & 13 & 6 & 7 & 7 & 12 & 6\end{array}$ $\begin{array}{llllllllllllllllllllll}\mathrm{m}^{2} & 10 & 10 & 4 & 5 & 10 & 10 & 3 & 4 & 2 & 10 & 3 & 10 & 10 & 10 & 5 & 2 & 10 & 2 & 2 & 10 & 10\end{array}$

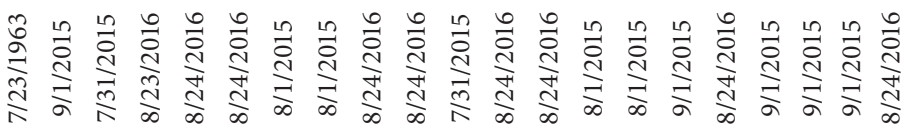

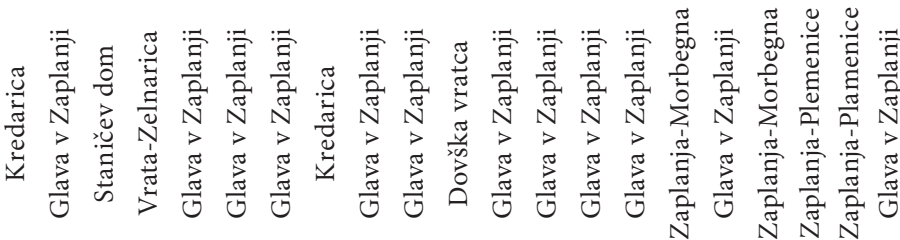

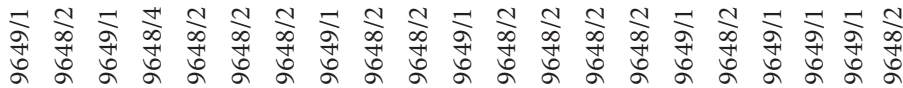

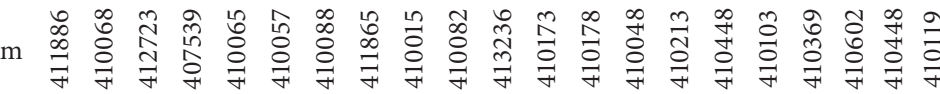

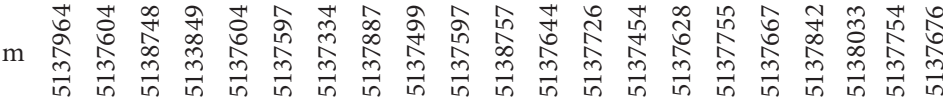

Diagnostic species of the syntaxa (Diagnostične vrste sintaksonov)

PS Potentilla nitida

TR1 Crepis terglouensis

PC Eritrichium nanum

TR1 Alyssum ovirense

PC Minuartia cherlerioides

AA Cerastium uniflorum

TR1 Saxifraga sedoides

Cfir Saxifraga exarata subsp. carniolica

TR1 Thlaspion rotundifolii Papaver julicum Thlaspi cepeaefolium (T. rotundifolium, Noccaea rotundifolia)

TR2 Thlaspieetalia rotundifolii Poa minor

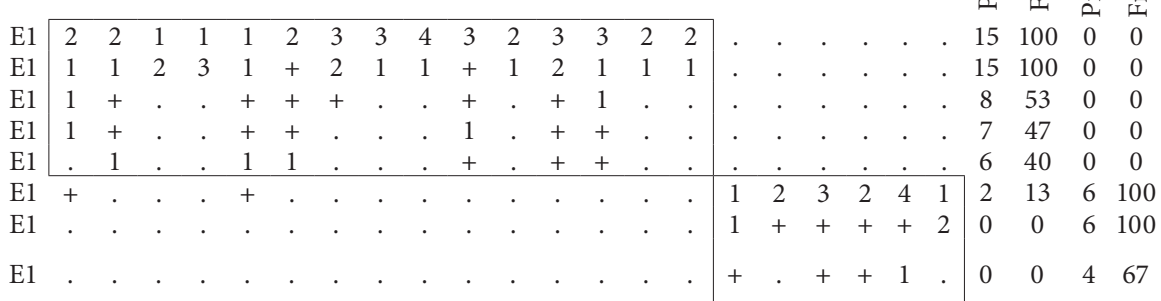

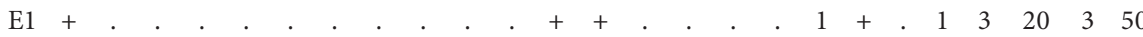

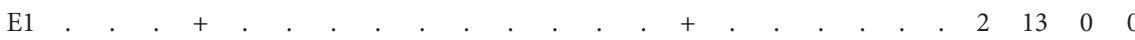

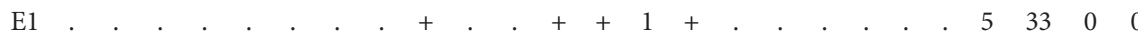


Number of relevé (Zaporedna številka popisa)

Achillea atrata

Cerastium carinthiacum subsp. carinthiacum

Moehringia ciliata

Armeria alpina

AC Arabidetalia caeruleae Salix retusa

Salix serpyllifolia

CD Carex capillaris

Carex ornithopodoides

Carex parviflora

SH Sagina saginoides

Saxifraga androsacea

DH Sesleria ovata

TR3 Thlaspietea rotundifolii

Taraxacum alpinum

Saxifraga oppositifolia s.str.

Linaria alpina

Festuca nitida

Arabis alpina

PC Potentilletalia caulescentis

Valeriana elongata

Festuca alpina

Campanula cochleariifolia

Saxifraga paniculata

Petrocallis pyrenaica

Cfir Caricion firmae

Minuartia verna

Silene acaulis

Carex firma

Minuartia sedoides

Phyteuma sieberi

Sesleria sphaerocephala

Gentiana terglouensis

Festuca quadriflora

Saussurea pygmaea

Veronica aphylla

OE Oxytropido-Elynion

Arenaria ciliata

Erigeron uniflorus

Lloydia serotina

ES Elyno-Seslerietea

PAT Poa alpina

Myosotis alpestris

Polygonum viviparum

ML Mosses (Mahovi)

Syntrichia norvegica

Bryum sp.

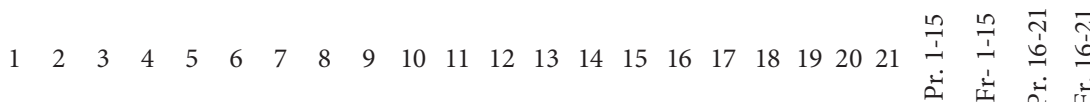

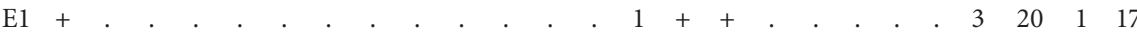

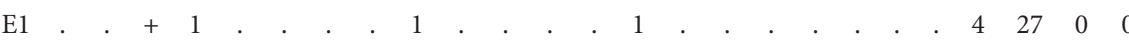

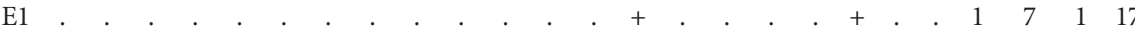

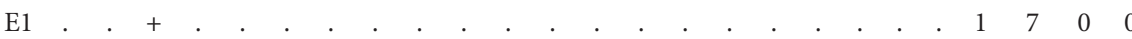

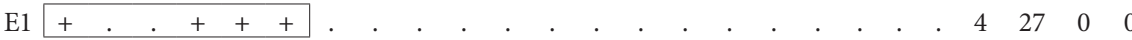

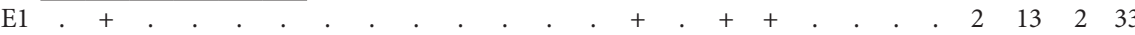

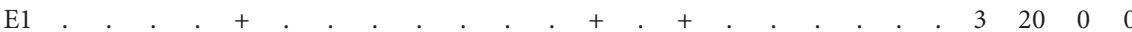

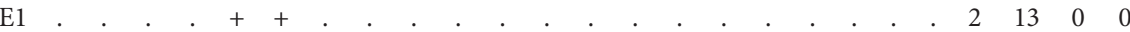

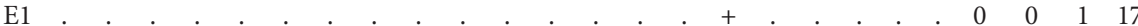

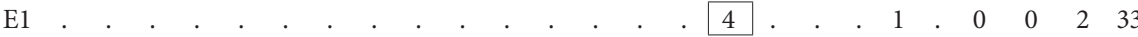

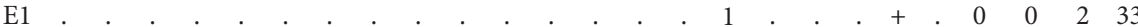

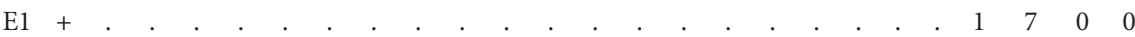

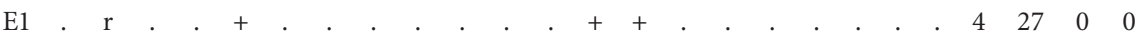

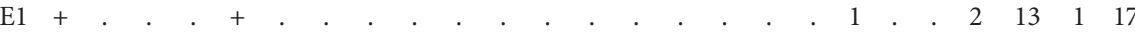

E1 . . . . . . . . . . . + . . . . . . . . . 117000

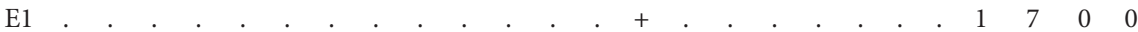

$\mathrm{E} 1 \ldots \ldots+\ldots+\ldots 0017$

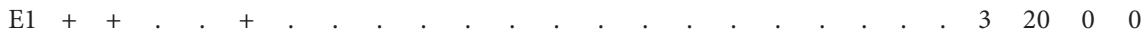

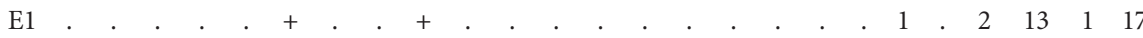

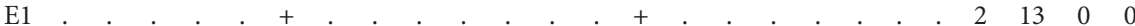

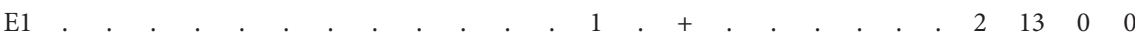

E1 . . . . . . . . . . + . . . . + . 2 . 131317

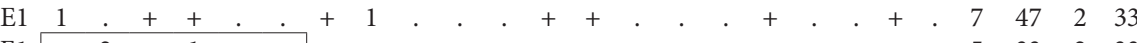

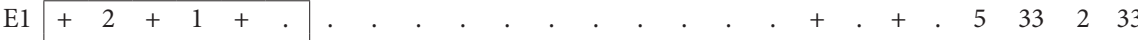

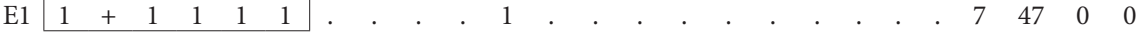

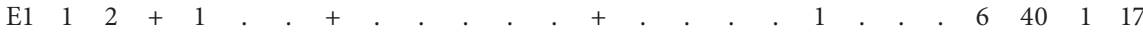

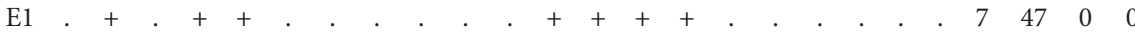

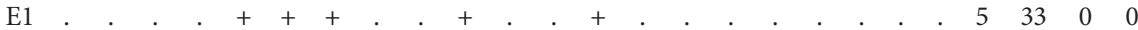

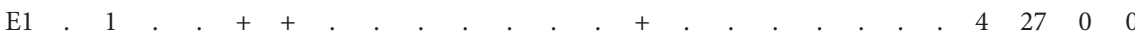

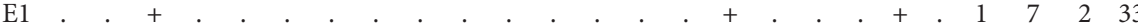

E1 . . . . . . . . . . . . . . . . .

E1 . . . . . . . . . . . . . . . . . . . . . . .

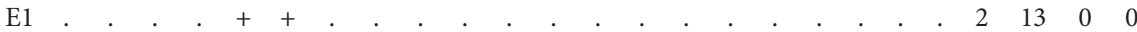

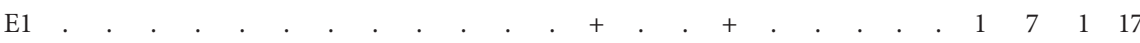

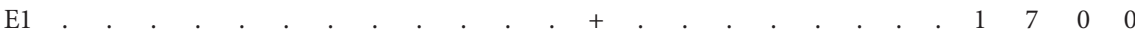

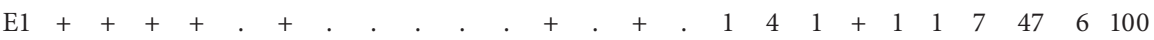

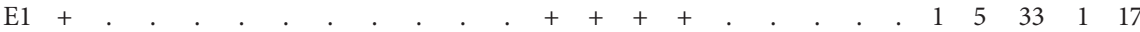

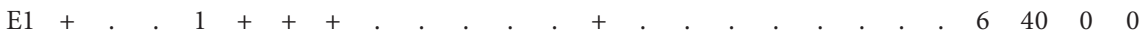

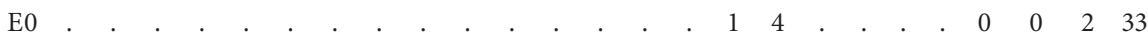

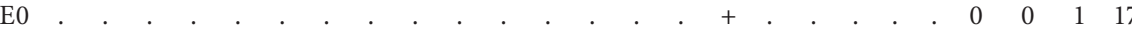

Legend - Legenda

ID Igor Dakskobler

BZ Branko Zupan

TW Tone Wraber

Gr Gravel - grušč

Li Lithosol - kamnišče

Pr. Presence (number of relevés in which the species is presented) - število popisov, v katerih se pojavlja vrsta

Fr. Frequency in $\%$ - frekvenca $v \%$

Re Rendzina - rendzina

PS Physoplexido-Saxifragion petraeae

AA Androsacion alpinae

CD Caricetalia davallianae

SH Salicetea herbaceae

DH Drabion hoppeanae

PAT Poo alpinae-Trisetetalia 
Table 2: Groups of diagnostic species in the stands of syntaxa Crepido terglouensis-Potentilletum nitidae (CtPn) and Saxifrago carniolicae-Cerastietum uniflorae ( $\mathrm{ScCu}$ ), relative frequencies

Preglednica 2: Skupine diagnostičnih vrst v sestojih sintaksonov Crepido terglouensis-Potentilletum nitidae (CtPn) and Saxifrago carniolicae-Cerastietum uniflorae $(\mathrm{ScCu})$, relativne frekvence

Successive number (Zaporedna številka)

Sign for syntaxa (Oznaka sintaksona)

Number of relevés (Število popisov)

Arabidetalia caeruleae

Thlaspion rotundifolii

Thlaspieetalia rotundifolii

Thlaspietea rotundifolii

Potentilletalia caulescentis

Caricion firmae

Oxytropido-Elynion

Elyno-Seslerietea

Moses (Mahovi)

Total (Skupaj)

$\begin{array}{cc}1 & 2 \\ \mathrm{CtPn} & \mathrm{ScCu} \\ 15 & 6 \\ \mathbf{8 , 3 0 4} & \mathbf{2 6 , 0 1} \\ \mathbf{1 6 , 0 7} & \mathbf{1 8 , 0 1} \\ \mathbf{8 , 3 3 3} & 4,002 \\ 4,762 & 4,002 \\ \mathbf{2 3 , 8 1} & 4,002 \\ 25,6 & \mathbf{2 4 , 0 1} \\ 2,381 & 2,001 \\ 10,71 & \mathbf{1 4 , 0 1} \\ 0 & 4,002 \\ 100 & 100\end{array}$


Table 3: Communities with Geum reptans in the Triglav Mts.

Preglednica 3: Združbi z vrsto Geum reptans v Triglavskem pogorju

Number of relevé (Zaporedna številka popisa)

Database number of relevé (Delovna številka popisa)

Author of the relevé (Avtor popisa)

Elevation in $\mathrm{m}$ (Nadmorska višina $\mathrm{v} \mathrm{m})$

Aspect (Lega)

Slope in degrees (Nagib v stopinjah)

Parent material (Matična podlaga)

Soil (Tla)

Stoniness in \% (Kamnitost v \%)

Cover of herb layer in \% (Zastiranje zeliščne plasti v \%):

Number of species (Število vrst)

Relevé area (Velikost popisne ploskve)

Date of taking relevé (Datum popisa)

Locality (Nahajališče)

Quadrant (Kvadrant)

Coordinate GK Y (D-48)

Coordinate GK X (D-48)

AA Androsacion alpinae

Geum reptans

AC Arabidetalia caeruleae

Doronicum glaciale

Galium noricum

Salix retusa

TR1 Thlaspion rotundifolii

Thlaspi cepeaefolium (T. rotundifolium, Noccaea rotundifolia)

Alyssum ovirense

Papaver julicum

Saxifraga sedoides

TR2 Thlaspieetalia rotundifolii

Achillea atrata

Moehringia ciliata

Festuca nitida

Poa minor

TR3 Thlaspietea rotundifolii

Saxifraga oppositifolia s.str.

Taraxacum alpinum agg.

Arabis alpina

PS Physoplexido-Saxifragion petraeae

Potentilla nitida

Paederota lutea

Saxifraga crustata

Saxifraga squarrosa

PC Potentilletalia caulescentis

Saxifraga paniculata

Festuca alpina

Campanula cochleariifolia

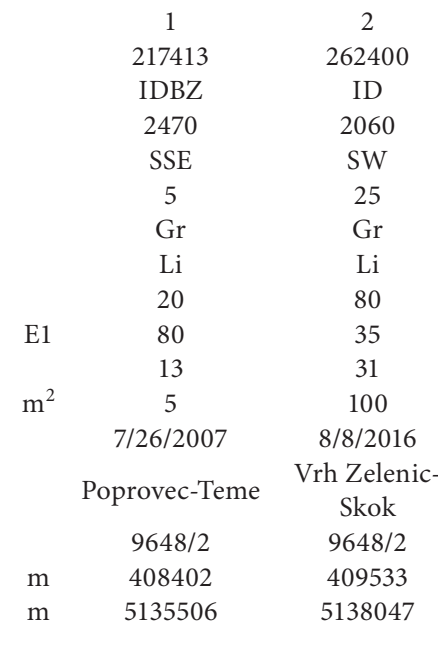

E1

4

CA Caricion austroalpinae

Arabis vochinensis

Cfir Caricion ferrugineae

Gentiana pumila

OE Oxytropido-Elynion

Arenaria ciliata

Cfir Caricion firmae

Minuartia verna

Carex firma

Dryas octopetala

Pedicularis rostratocapitata

Phyteuma sieberi

Silene acaulis

\begin{tabular}{|c|c|c|}
\hline E1 & + & . \\
\hline E1 & + & . \\
\hline E1 & . & + \\
\hline E1 & 1 & . \\
\hline E1 & . & + \\
\hline E1 & . & + \\
\hline E1 & . & + \\
\hline E1 & + & 1 \\
\hline E1 & + & . \\
\hline E1 & . & + \\
\hline E1 & . & + \\
\hline E1 & . & + \\
\hline E1 & . & + \\
\hline E1 & + & + \\
\hline E1 & . & + \\
\hline E1 & . & + \\
\hline E1 & . & + \\
\hline E1 & . & + \\
\hline E1 &. & + \\
\hline
\end{tabular}


Number of relevé (Zaporedna številka popisa)

ES Elyno-Seslerietea

Polygonum viviparum

Aster bellidiastrum

Pedicularis verticillata

Achillea clavenae

MC Montio-Cardaminetea

Saxifraga aizoides

PAT Poo alpinae-Trisetetalia

Poa alpina

Legend - Legenda

ID Igor Dakskobler

BZ Branko Zupan

Gr Gravel - grušč

Li Lithosol - kamnišče
1

2

Pr.

E1

E1

E1

E1

E1

E1

$+$
1

1
+

$+$

$+$

1

1
1

1

1

1
1

1

2 
Appendix 1: Synthetic table of communities with Potentilla nitida and/or Crepis terglouensis in Eastern and Southeastern Alps Dodatek 1: Sintezna tabela združb z vrstama Potentilla nitida in/ali Crepis terglouensis v Vzhodnih in Jugovzhodnih Alpah

Successive number (Zaporedna številka)

Number of relevés (Število popisov)

Sign for syntaxa (Oznaka sintaksonov)

TR1 Thlaspion rotundifolii

Crepis terglouensis

Galium megalospermum

Arabis bellidifolia subsp. bellidifolia

Pritzelago alpina subsp. alpina (Hutchinsia alpina)

Saxifraga aphyla

Thlaspi cepeaefolium (T. rotundifolium, Noccaea rotundifolia)

Alyssum ovirense

Papaver julicum

Saxifraga sedoides

Festuca rupicaprina

TR2 Thlaspietalia rotundifolii

Poa cenisia

Poa minor

Linaria alpina

Doronicum grandiflorum

Cerastium carinthiacum

Achillea atrata

Armeria alpina

Moehringia ciliata

Sedum atratum

AC Arabidetalia caeruleae

Salix serpyllifolia

MC Saxifraga aizoides

Ranunculus alpestris

Salix retusa

CD Carex capillaris

AA Cerastium uniflorum

Carex ornithopodoides

DH Sesleria ovata

JT Carex fuliginosa

Ranunculus traunfellneri

Doronicum glaciale

Salix reticulata

Trifolium pallescens

TR3 Thlaspietea rotundifolii

Athamantha cretensis

Trisetum distichophyllum

Gypsophila repens

Silene vulgaris subsp. glareosa

Taraxacum alpinum

Saxifraga oppositifolia s.str.

Festuca nitida

Pritzelago alpina subsp. brevicaulis

PS Physoplexido-Saxifragion petraeae

Potentilla nitida

Saxifraga squarrosa

Saxifraga crustata

Campanula zoysii

Paederota lutea

PC Potentilletalia caulescentis

Campanula cochleariifolia

Eritrichium nanum

Minuartia cherlerioides

Valeriana elongata

Festuca alpina

Petrocallis pyrenaica

\section{1}

25

CT

2
15
CTPN

3

33

PN

4
1
CTPN

E1

E1

E1

E1

E1

E1

E1

E1

E1

E1

E1

E1

E1

E1

E1

E1

E1

E1

E1

E1

E1

E1

E1

E1

E1

E1

E1

E1

E1

E1

E1

E1

E1

E1

E1

E1

E1

E1

E1

E1

E1

E1

E1

E1

E1

$\begin{array}{ccc}100 & 100 \\ 76 & \cdot \\ 52 & . & \\ 44 & \cdot & \\ 12 & . & \\ 8 & 13 & \\ . & 47 & 15 \\ . & 20 & 12 \\ . & . & 15 \\ . & . & 8\end{array}$

$\begin{array}{cc}. & 1 \\ . & . \\ 3 & . \\ . & . \\ . & . \\ 15 & \text {. } \\ 12 & \text {. } \\ 15 & \text {. } \\ 8 & \text {. }\end{array}$

$\begin{array}{cccc}24 & . & . & . \\ 16 & 33 & . & . \\ 8 & 7 & 6 & + \\ 4 & . & . & . \\ . & 27 & 3 & + \\ . & 20 & 3 & . \\ . & 7 & . & + \\ . & 7 & . & . \\ . & . & 3 & .\end{array}$

$$
16
$$$$
13
$$$$
15
$$

$$
1
$$$$
12
$$$$
4
$$$$
\text { . }
$$$$
\text { . }
$$$$
\text { (n) }
$$$$
\text { (n) }
$$$$
\text { (2) }
$$$$
27
$$$$
\begin{aligned}
& 27 \\
& 20 \\
& 13
\end{aligned}
$$$$
\begin{aligned}
& 13 \\
& 13
\end{aligned}
$$$$
\begin{gathered}
13 \\
7
\end{gathered}
$$$$
\text { . }
$$$$
\text { . }
$$$$
\text { . }
$$$$
36
$$$$
\begin{aligned}
& 36 \\
& 12
\end{aligned}
$$$$
\begin{gathered}
12 \\
8
\end{gathered}
$$$$
8
$$$$
\text { . }
$$$$
\text { . }
$$$$
\text { . }
$$$$
13
$$$$
\begin{gathered}
7 \\
7
\end{gathered}
$$$$
\text { . }
$$$$
100
$$$$
2
$$$$
\text { . }
$$ 
Successive number (Zaporedna številka)

Saxifraga paniculata

Draba tomentosa

Saxifraga exarata subsp. carniolica

Potentilla clusiana

Arabis bellidifolia subsp. stellulata

Saxifraga burseriana

Androsace helvetica

Primula auricula

Dianthus sylvestris

CF Caricion ferrugineae

Viola biflora

Heliosperma pusillum

Carex ferruginea

Cfir Caricion firmae

Festuca quadriflora

Carex firma

Dryas octopetala

Minuartia verna

Silene acaulis

Minuartia sedoides

Crepis kerneri

Saxifraga caesia

Phyteuma sieberi

Sesleria sphaerocephala

Gentiana terglouensis

Saussurea pygmaea

Helianthemum alpestre

Gentiana orbicularis

Salix alpina

Oxytropis neglecta

Pedicularis rostratocapitata

Carex rupestris

Pedicularis rosea

Veronica aphylla

OE Oxytropido-Elynion

Arenaria ciliata

Lloydia serotina

Erigeron uniflorus

ES Elyno-Seslerietea

Polygonum viviparum

Sesleria caerulea

Euphrasia salisburgensis

Carex sempervirens

Pedicularis oederi

Galium anisophyllon

PaT Poa alpina

Myosotis alpestris

Draba aizoides

Erigeron glabratus

Achillea clavenae

Leontopodium alpinum

Agrostis alpina

Aster bellidiastrum

EP Erico-Pinetea

Asperula aristata

Rhodothamnus chamaecistus

O Other species (Druge vrste)

Saxifraga sp.

Thymus sp.

$\begin{array}{lcccc} & 1 & 2 & 3 & 4 \\ \text { E1 } & . & 13 & 61 & . \\ \text { E1 } & . & . & 45 & . \\ \text { E1 } & . & . & 24 & . \\ \text { E1 } & . & . & 21 & 1 \\ \text { E1 } & & . & 18 & . \\ \text { E1 } & . & . & 12 & . \\ \text { E1 } & . & . & 6 & . \\ \text { E1 } & . & . & 3 & . \\ \text { E1 } & . & . & 3 & .\end{array}$

ML Mosses and lichens (Mahovi in lišaji)

Tortella tortuosa

Distichum capillaceum

\begin{tabular}{|c|c|c|c|}
\hline E1 & 40 & . & 3 \\
\hline E1 & 12 & . & . \\
\hline E1 & 4 & . & . \\
\hline E1 & 76 & 7 & 6 \\
\hline E1 & 56 & 47 & 45 \\
\hline E1 & 32 & . & 3 \\
\hline E1 & 24 & 47 & 61 \\
\hline E1 & 24 & 33 & 30 \\
\hline E1 & 12 & 40 & 82 \\
\hline E1 & 8 & . & 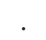 \\
\hline E1 & 8 & . & 3 \\
\hline E1 & . & 47 & . \\
\hline E1 & . & 33 & 70 \\
\hline E1 & . & 27 & 52 \\
\hline E1 & . & 7 & 6 \\
\hline E1 & . & . & 18 \\
\hline E1 & . & . & 12 \\
\hline E1 & . & . & 9 \\
\hline E1 & . & . & 6 \\
\hline E1 & . & . & 6 \\
\hline E1 & . & . & 3 \\
\hline E1 & . & . & 3 \\
\hline E1 & . & . & 3 \\
\hline E1 & & 13 & 33 \\
\hline E1 & . & 7 & 15 \\
\hline E1 & . & 7 & 3 \\
\hline E1 & 40 & 40 & 39 \\
\hline E1 & 28 & . & 9 \\
\hline E1 & 12 & . & 3 \\
\hline E1 & 4 & . & 3 \\
\hline E1 & 4 & . & . \\
\hline E1 & 4 & . & . \\
\hline E1 & . & 47 & 55 \\
\hline E1 & . & 33 & 3 \\
\hline E1 & . & 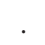 & 33 \\
\hline E1 & . & . & 18 \\
\hline E1 & . & . & 15 \\
\hline E1 & . & . & 6 \\
\hline E1 & . & . & 3 \\
\hline E1 & . & . & 3 \\
\hline E1 & 8 & . & 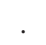 \\
\hline E1 & . & . & 3 \\
\hline E1 & . & . & 3 \\
\hline E1 & . & . & 3 \\
\hline E0 & 8 & $\cdot$ & . \\
\hline E0 & 8 & & \\
\hline
\end{tabular}


Successive number (Zaporedna številka)

Ctenidium molluscum

Orthotecium rufescens

Cladonia sp.

Pohlia cruda

Encalypta streptocarpa

Hypnum bambergeri

Ctenidium procerrimum

Legend - Legenda

CT Crepidetum terglouensis (Eggensbeger 1994, Table 6, Columns 85-111)

CTPN Crepido-Potentilletum nitidae, this article, Table 1, Coliumns 1-15

PN Potentilletum nitidae, T. Wraber 1972, Table 3

CTPN Crepido-Potentilletum nitidae, Sutter (1969: 353)

MC Montio-Cradaminetea

CD Caricetalia davallianae

AA Androsacion alpinae

DH Drabion hoppeanae

PAT Poo alpinae-Trisetetalia

JT Juncetea trifidi

Appendix 2: Communities with Cerastium uniflorum and Saxifraga sedoides in SE Alps Preglednica 2: Združbi z vrstama Cerastium uniflorum in Saxifraga sedoides v JV Alpah

Successive number (Zaporedna številka)

Number of relevés (Število popisov)

Sign for syntaxa (Oznaka sintaksonov)

AC Arabidetalia caeruleae

AA Cerastium uniflorum

MC Saxifraga stellaris subsp. alpigena Soldanella minima

AA Doronicum clusii Ranunculus alpestris

DH Draba hoppeana Salix serpyllifolia Saxifraga androsacea

$\mathrm{SH}$ Sagina saginoides Carex parviflora

\begin{tabular}{|c|c|c|}
\hline & 1 & 2 \\
\hline & 15 & 6 \\
\hline & Ss & $\mathrm{ScSu}$ \\
\hline E1 & 47 & 100 \\
\hline E1 & 13 & \\
\hline E1 & 13 & . \\
\hline E1 & 7 & . \\
\hline E1 & 7 & . \\
\hline E1 & 7 & . \\
\hline E1 & . & 33 \\
\hline E1 & . & 33 \\
\hline E1 & . & 33 \\
\hline E1 & . & 17 \\
\hline E1 & 100 & 100 \\
\hline E1 & 60 & . \\
\hline E1 & 53 & . \\
\hline E1 & 27 & . \\
\hline E1 & 20 & . \\
\hline E1 & . & 50 \\
\hline E1 & 67 & 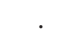 \\
\hline E1 & 60 & 17 \\
\hline E1 & 47 & 17 \\
\hline E1 & 20 & 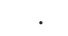 \\
\hline E1 & 13 & . \\
\hline E1 & . & 17 \\
\hline
\end{tabular}


TR3 Thlaspietea rotundifolii

Saxifraga oppositifolia s.str.

Pritzelago alpina subsp. brevicaulis

Taraxacum alpinum

PC Potentilletalia caulescentis

Festuca alpina

Campanula cochleariifolia

Androsace hausmanii

Phyteuma sieberi

Valeriana elongata

Cystopteris regia

Petrocallis pyrenaica

Saxifraga exarata subsp. carniolica

Minuartia sedoides

Sesleria sphaerocephala

Minuartia verna

Silene acaulis

OE Erigeron uniflorus

Festuca quadriflora

Veronica aphylla

ES Elyno-Seslerietea

PAT Poa alpina

Polygonum viviparum

CF Heliosperma pusillum

Myosotis alpestris

Mosses and lichens (Mahovi in lišaji)

Musci spp.

Grimmia sp.

Marchantia sp.

Syntrichia norvegica

Bryum sp.

$\begin{array}{lcc}\text { E1 } & 20 & 17 \\ \text { E1 } & 13 & . \\ \text { E1 } & 7 & 33 \\ \text { E1 } & 7 & 33 \\ \text { E1 } & 7 & 17 \\ \text { E1 } & \cdot & 33 \\ \text { E1 } & \cdot & 17 \\ & \cdot & \\ \text { E1 } & 27 & 100 \\ \text { E1 } & 13 & . \\ \text { E1 } & 7 & . \\ \text { E1 } & . & 17\end{array}$

Legend - Legenda

Ss Saxifragetum sedoidis, E. \& S. Pignatti, 2016, Association Table 9.2

$\mathrm{ScCu}$ Saxifrago carniolicae-Cerastietum uniflorae, this article

MC Montio-Cradaminetea

CD Caricetalia davallianae

AA Androsacion alpinae

DH Drabion hoppeanae

PAT Poo alpinae-Trisetetalia

SH Salicetea herbaceae

CF Caricion ferrugineae 